\title{
Modules of finite projective dimension with negative intersection multiplicities
}

Sankar P. Dutta ${ }^{1}$, M. Hochster ${ }^{1,2}$, and J.E. McLaughlin

${ }^{1}$ The University of Pennsylvania, Philadelphia, PA

and the Institute for Advanced Study, Princeton, NJ 08540, USA

${ }^{2}$ The University of Michigan, Ann Arbor, MI 48109, USA

\section{Introduction}

Let $X_{i}, 1 \leqq i \leqq 4$, be indeterminates over a field $K$. Let $S=K\left[X_{1}, X_{2}, X_{3}, X_{4}\right]$ and $q=\sum_{i=1}^{4} X_{i} S$. Throughout this paper $R$ shall denote the local three-dimensional hypersurface

$$
S_{q} /\left(X_{1} X_{4}-X_{2} X_{3}\right)
$$

Let $x_{i}$ be the image of $X_{i}$ in $R$.

The main object of this paper is to study modules of finite length and finite projective dimension over $R$. One consequence of our study will be counterexamples to several conjectures concerning the behavior of intersection multiplicities for modules of finite projective dimension.

Let " $l$ " denote length. If $S$ is a Noetherian ring, $M, N$ are finitely generated $S$-modules, $l\left(M \otimes_{S} N\right)$ is finite, and $d=p d_{S} M<\infty$, we may define, following Serre $[S]$

$$
\chi_{S}(M, N)=\chi(M, N)=\sum_{i=0}^{d}(-1)^{i} l\left(\operatorname{Tor}_{i}^{S}(M, N)\right) .
$$

When $S$ is regular, $p d_{S} M \leqq \operatorname{dim} S$ automatically. For regular local rings $(S, m)$ such that the m-adic completion $\hat{S}$ of $S$ is a formal power series ring over a field or discrete valuation ring, Serre proved that

1) if $\operatorname{dim} M+\operatorname{dim} N<\operatorname{dim} S$, then $\chi(M, N)=0$.

2) if $\operatorname{dim} M+\operatorname{dim} N=\operatorname{dim} S$, then $\chi(M, N)>0$.

Serre proved that the hypothesis $l\left(M \otimes_{S} N\right)<\infty$ implies $\operatorname{dim} M$ $+\operatorname{dim} N \leqq \operatorname{dim} S$ for any regular local ring $S$, and he conjectured that 1) and 2) remain valid for an arbitrary regular local ring $S$. This is still an open question.

The first two authors were supported in part by grants from the National Science Foundation

The second author was a Guggenheim Fellow while this research was being carried out 
See $[\mathrm{W}],[\mathrm{S}],\left[\mathrm{H}_{1}\right],\left[\mathrm{H}_{2}\right],\left[\mathrm{H}_{3}\right],[\mathrm{M}],[\mathrm{L}],\left[\mathrm{PS}_{2}\right],\left[\mathrm{D}_{1}\right],\left[\mathrm{D}_{2}\right],\left[\mathrm{D}_{3}\right],\left[\mathrm{D}_{4}\right]$, and $\left[\mathrm{D}_{5}\right]$ for additional background.

It is natural to ask whether 1) and 2) continue to hold when the regularity condition on $S$ is dropped, but it is required instead that $p d_{S} M$ be finite. Questions of this sort arise naturally in trying to study 1) and 2) even in the regular case. Some partial results have been obtained: see $\left[\mathrm{PS}_{2}\right],\left[\mathrm{D}_{1}\right],\left[\mathrm{D}_{4}\right]$ and $\left[\mathrm{D}_{5}\right]$.

However, in the sequel we shall exhibit modules $M$ of finite length and finite projective dimension over the ring $R$ described earlier such that 1) and 2) both fail. This disproves a large number of conjectures: see $\$ 7$. It also follows that the Grothendieck group of modules of finite length and finite projective dimension over $R$ is not generated by the classes of the modules of the form $R /(u, v, w) R$, where $u, v, w$ is an $R$-sequence, nor even, in fact, by the classes of those modules of finite length and finite projective dimension which are extended from unramified regular rings. This remains true even if we apply $\otimes_{\mathbf{z}} \mathbf{Q}$ to the Grothendieck group. This answers negatively questions raised in $\left[\mathrm{H}_{1}\right]$.

We shall return to an examination of the consequences of our investigation in $\S 7$.

It is worth mentioning that our "counterexample" modules are killed by $I$ $=\mathrm{m}^{3}+\left(x_{2}, x_{4}\right) \mathrm{m}$, where $\mathrm{m}=\sum_{i=1}^{4} x_{i} R$, and that the counterexample of least length killed by this ideal has length 15 . That is, there is a module $M$ of finite projective dimension killed by $I$ of length 15 such that

$$
\chi(M, R / P)=-1
$$

where $P=x_{1} R+x_{2} R$. (However if $M$ has finite projective dimension, $l(M)<15$, and $M$ is killed by $I$ then $\chi(M, N)=0$ for all finitely generated $R$-modules $N$ such that $\operatorname{dim} N<3$.)

We shall also see that these "minimal" counterexamples of length 15 have no nonzero proper submodules (respectively, quotients) of finite projective dimension.

The intense study of the behavior of $\chi(R / P, M)$ undertaken here was motivated by an unpublished argument of S.P. Dutta which showed that the generalized Serre conjecture for $R / P$ and an arbitrary module $M$ of finite projective dimension implies the corresponding conjecture over a hypersurface when both modules have finite projective dimension. Moreover, his argument was also valid for hypersurfaces of the form $V\left[\left[X_{1}, \ldots, X_{n}\right]\right] /(F)$ with $V$ a discrete valuation ring, with $R$ replaced by $R_{1}=V\left[X_{1}, X_{2}, X_{3}, X_{4}\right] /\left(X_{1} X_{4}-X_{2} X_{3}\right)$ localized at $\mathrm{m}=\mathrm{m}_{v}+\left(X_{i}\right)$. Thus, the generalized Serre conjecture for $R_{1}$ would have implied the original Serre conjecture for ramified regular rings.

The original Serre conjecture remains an open question, as does the generalized form if one assumes that both modules have finite projective dimension. (See 'Note added in proof', at the end of the references.)

\section{Finite length $R$-modules}

To give a module $M$ of length $n$ over $R$ is the same as to give the following data: 
(2.1) 1) An $n$-dimensional $K$-vector space $M$.

2) Four $K$-endomorphisms $\phi_{1}, \phi_{2}, \phi_{3}, \phi_{4}$ of $M$ such that
a) $\phi_{i} \phi_{j}=\phi_{j} \phi_{i}, \quad 1 \leqq i, j \leqq 4$
b) $\phi_{1} \phi_{4}=\phi_{2} \phi_{3}$
c) $\phi_{i}$ is nilpotent, $\quad 1 \leqq i \leqq 4$.

Given a module $M$, we obtain a $K$-vector space by restriction of scalars, and we let $\phi_{i}$ be the map $u \mapsto x_{i} u, 1 \leqq i \leqq 4$. Conversely, given $M$ and the $\phi_{i}$, conditions 1) and $2 \mathrm{a}), 2 \mathrm{~b}$ ) suffice to guarantee that there is a unique $K\left[x_{1}, x_{2}, x_{3}, x_{4}\right]$-module structure on $M$ extending its $K$-vector space structure and such that multiplication by $x_{i}$ is the map $\phi_{i}$. Condition $2 \mathrm{c}$ ) is then equivalent to the condition that $M$ be an $R$-module, for this will be the case if and only if each $x_{i}$ acts nilpotently on $M$.

Given $\phi_{1}, \phi_{2}, \phi_{3}, \phi_{4}: M \rightarrow M$ they determine an endomorphism $\phi: M \oplus M$ $\rightarrow M \oplus M$ by

$$
\phi\left(u \oplus u^{\prime}\right)=\left(\phi_{1}(u)+\phi_{2}\left(u^{\prime}\right)\right) \oplus\left(\phi_{3}(u)+\phi_{4}\left(u^{\prime}\right)\right),
$$

i.e. $\phi$ is represented by the $2 \times 2$ matrix

$$
\left[\begin{array}{ll}
\phi_{1} & \phi_{2} \\
\phi_{3} & \phi_{4}
\end{array}\right]
$$

over $\operatorname{End}_{R} M$. Note that matrices act on the left.

Thus, there is a one-one correspondence between $R$-module structures on the $K$-vector space $M$ and the subset of elements

$$
\phi=\left[\begin{array}{ll}
\phi_{1} & \phi_{2} \\
\phi_{3} & \phi_{4}
\end{array}\right] \in \operatorname{End}_{R}(M \oplus M)
$$

such that the $\phi_{i}$ satisfy the conditions $2 \mathrm{abc}$ ) listed in (2.1).

We make the following notational convention: if $\phi \in \operatorname{End}_{R}(M \oplus M)$ and

$$
\phi=\left[\begin{array}{ll}
\phi_{1} & \phi_{2} \\
\phi_{3} & \phi_{4}
\end{array}\right] \quad \text { then } \phi^{\vee}=\left[\begin{array}{rr}
\phi_{4} & -\phi_{2} \\
-\phi_{3} & \phi_{1}
\end{array}\right] .
$$

One readily checks that $\phi^{\vee \vee}=\phi$ and that conditions $2 \mathrm{a}$ ), 2b) are equivalent to

$$
\phi \phi^{\vee}=\phi^{\vee} \phi=0 .
$$

Moreover, $\phi$ satisfies $2 \mathrm{abc}) \Leftrightarrow \phi^{\vee}$ satisfies $2 \mathrm{abc}$ ).

Of course, if $l(M)\left(=\operatorname{dim}_{K} M\right)=n$ we can choose a basis and so identify $M \cong K^{n}$. By iterating this basis we get an isomorphism $M \oplus M \cong K^{2 n}$.

The $\phi_{i}$ then correspond to $n \times n$ matrices $A_{i}$ satisfying the analogues of conditions $2 \mathrm{abc}$ ) listed in (2.1), and $\phi$ to a matrix

$$
A=\left[\begin{array}{ll}
A_{1} & A_{2} \\
A_{3} & A_{4}
\end{array}\right]
$$


which may be thought of as a $2 \times 2$ matrix whose entries are $n \times n$ matrices, or as a single $2 n \times 2 n$ matrix. We shall usually take the second viewpoint always, when we deal with "rank". This ambiguity should not cause confusion. In this context

$$
A^{\vee}=\left[\begin{array}{rr}
A_{4} & -A_{2} \\
-A_{3} & A_{1}
\end{array}\right]
$$

We now fix some notation. Let $P=x_{1} R+x_{2} R\left(\cong x_{3} R+x_{4} R\right.$ as a module) and let $Q=x_{1} R+x_{3} R$ ( $\cong x_{2} R+x_{4} R$ as a module). Let

$$
X=\left[\begin{array}{ll}
x_{1} & x_{2} \\
x_{3} & x_{4}
\end{array}\right] \text { and } \quad X^{\vee}=\left[\begin{array}{rr}
x_{4} & -x_{2} \\
-x_{3} & x_{1}
\end{array}\right]
$$

(2.2) Lemma. a) The complexes

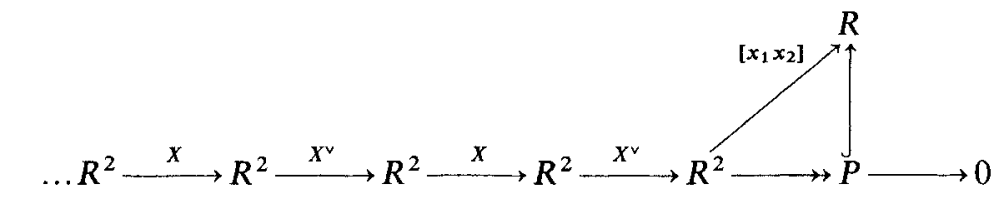

and

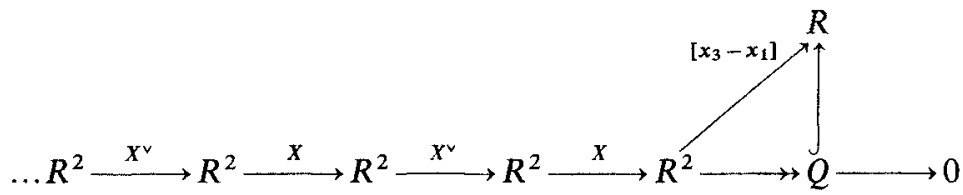

are minimal free resolutions of $P$ and $Q$, respectively, over $R$, periodic of period 2.

b) $P \cap Q=x_{1} R \cong R$.

Proof. a) It suffices to prove these results when " $R$ " is defined to be $K\left[X_{1}, X_{2}, X_{3}, X_{4}\right] /\left(X_{1} X_{4}-X_{2} X_{3}\right)$, without localizing, for they are "preserved" by localization, and we so redefine $R$ for the course of this proof.

To show the exactness of $R^{2} \stackrel{X^{v}}{\longrightarrow} R \longrightarrow P$ at the middle spot we must show that all relations on $x_{1}, x_{2}$ are spanned by $\left(x_{4},-x_{3}\right)$ and $\left(-x_{2}, x_{1}\right)$. But if $u x_{1}$ $+v x_{2}=0$ we have, lifting this to $K\left[X_{1}, X_{2}, X_{3}, X_{4}\right]$,

$$
U X_{1}+V X_{2}=H\left(X_{4} X_{1}-X_{2} X_{3}\right)
$$

where each capital letter (e.g. $U, V$ ) represents a lifting of the corresponding lower case letter. Equivalently,

$$
\left(U-H X_{4}\right) X_{1}=-\left(V+H X_{3}\right) X_{2}
$$

which implies $X_{2} \mid\left(U-H X_{4}\right)$, i.e. $U=H X_{4}+G X_{2}$. But then $V=-H X_{3}-G X_{1}$ and

$$
(u, v)=g\left(x_{2},-x_{1}\right)+h\left(x_{4},-x_{3}\right), \quad \text { as required. }
$$


The relations on $x_{3}, x_{4}$ or on the columns of $\left[\begin{array}{ll}x_{1} & x_{2} \\ x_{3} & x_{4}\end{array}\right]$ are the same, which shows the exactness of $R^{2} \stackrel{x^{\vee}}{\longrightarrow} R^{2} \stackrel{x}{\longrightarrow} R^{2}$ at the middle spot. Similarly, the relations on $x_{4},-x_{2}$ or $-x_{3}, x_{1}$ or on the columns of $\left[\begin{array}{rr}x_{4} & -x_{2} \\ -x_{3} & x_{1}\end{array}\right]$ are spanned by $\left(x_{1}, x_{3}\right)$ and $\left(x_{2}, x_{4}\right)$, which shows the exactness of $R^{2} \stackrel{x}{\longrightarrow} R^{2} \stackrel{x}{\longrightarrow} R^{2}$ at the middle spot. This proves that the first complex is a resolution. (Minimality is clear, since all the matrices have their entries in the ideal $\left(x_{1}, x_{2}, x_{3}, x_{4}\right)$.)

The same facts yield the exactness of the second complex as well. Q.E.D.

b) Suppose $r \in P \cap Q$, say $r=u x_{1}+v x_{2}=u^{\prime} x_{1}+w x_{3}$. Then $v x_{2}=\left(u^{\prime}-u\right) x_{1}$ $+w x_{3} \in\left(x_{1}, x_{3}\right)=Q$, a prime, so that, since $x_{2} \notin Q$, we have $v \in Q$, say $v=s x_{1}$ $+t x_{3}$. Then

$$
\begin{aligned}
r & =u x_{1}+\left(s x_{1}+t x_{3}\right) x_{2}=u x_{1}+s x_{2} x_{1}+t x_{2} x_{3} \\
& =u x_{1}+s x_{2} x_{1}+t x_{1} x_{4}=\left(u+s x_{2}+t x_{4}\right) x_{1} \in x_{1} R .
\end{aligned}
$$

But $x_{1} R \subset P \cap Q$ is clear. Q.E.D.

(2.3) Corollary. There are exact sequences:

$$
0 \rightarrow Q \rightarrow R^{2} \rightarrow P \rightarrow O \text { and } O \rightarrow P \rightarrow R^{2} \rightarrow Q \rightarrow O .
$$

Proof. In part a) of (2.2) the kernel of $R^{2} \rightarrow P$ is the column space (image) of $X^{\vee}$, i.e. $R\left[\begin{array}{r}x_{4} \\ -x_{3}\end{array}\right]+R\left[\begin{array}{r}-x_{2} \\ x_{1}\end{array}\right]$. Projection on the second coordinate gives an isomorphism of this module with $Q$. The argument for the second sequence is similar. Q.E.D.

Thus, each of $P$ and $Q$ is a first module of syzygies (or relations) for the other. Of course, eventual periodicity for minimal resolutions over a hypersurface is expected: see $[E]$.

(2.4) Proposition. Let $M$ be a finitely generated $R$-module. Let $P=\left(x_{1}, x_{2}\right)$ and $Q=\left(x_{1}, x_{3}\right)$, as above. Then the following conditions are equivalent:

1) $M$ has finite projective dimension.

2) For all sufficiently large $i, \operatorname{Tor}_{i}^{R}(M, K)=0$.

3) For all sufficiently large $i, \operatorname{Tor}_{i}^{R}(M, P)=0$.

4) For all sufficiently large $i, \operatorname{Tor}_{i}^{R}(M, Q)=0$.

5) For all $i \geqq 1$, $\operatorname{Tor}_{i}^{R}(M, P)=0$.

6) For all $i \geqq 1, \operatorname{Tor}_{i}^{R}(M, Q)=0$.

Proof. 1) $\Leftrightarrow 2$ ) is well known, while it is clear that $(2.3)$ implies 3$) \Leftrightarrow 4$ ), and that 1) implies both 3) and 4). We next show that 3) and 4) imply 2), which will show that 1), 2), 3), and 4) are equivalent.

If 3) (or 4)) holds, both hold. Then, from the exact sequence

$$
0 \rightarrow P \cap Q \rightarrow P \oplus Q \rightarrow P+Q \rightarrow 0
$$


and the fact that $P \cap Q \cong R$ (by (2.2b)), we conclude that, if $J=P+Q=x_{1} R$ $+x_{2} R+x_{3} R$, we have that $\operatorname{Tor}_{i}^{R}(M, J)=0$ for all sufficiently large $i$ and hence that $\operatorname{Tor}_{i}^{R}(M, R / J)=0$ for all sufficiently large $i$ as well. But $B=R / J \cong K\left[x_{4}\right]_{\left(x_{4}\right)}$ so that $0 \rightarrow B \stackrel{x_{4}}{\rightarrow} B \rightarrow K \rightarrow 0$ is exact and so $\operatorname{Tor}_{i}^{R}(M, K)=0$ for all sufficiently large $i$.

Thus, 1), 2), 3), and 4) are equivalent. By Corollary (2.3), $\operatorname{Tor}_{i}^{R}(M, P)$ $=\operatorname{Tor}_{i+2}^{R}(M, P)$ for $i \geqq 1$ and the same holds with $Q$ replacing $P$. Thus 3$) \Rightarrow 5$ ) and 4$) \Rightarrow 6$ ), while the converse implications are clear. Hence, all six conditions are equivalent. Q.E.D.

(2.5) Theorem. Let $M$ be an R-module of finite length $n$ and let $A$ be a corresponding $2 n \times 2 n$ matrix. Then $p d_{R} M<\infty \Leftrightarrow \operatorname{rank} A+\operatorname{rank} A^{\vee}=2 n$.

Proof. Consider the resolution of $Q$ given in (2.2a). If we drop the augmentation and apply $M \otimes_{R}$ we get

$$
\cdots \longrightarrow M^{2} \stackrel{X^{v}}{\longrightarrow} M^{2} \stackrel{x}{\longrightarrow} M^{2} \stackrel{X^{v}}{\longrightarrow} M^{2} \stackrel{x}{\longrightarrow} M^{2} \longrightarrow 0,
$$

where $M^{2}=M \oplus M$. If we identify $M^{2} \cong K^{2 n}$ this becomes:

$$
\ldots \longrightarrow K^{2 n} \stackrel{A^{\vee}}{\longrightarrow} K^{2 n} \stackrel{A}{\longrightarrow} K^{2 n} \stackrel{A^{\vee}}{\longrightarrow} K^{2 n} \stackrel{A}{\longrightarrow} K^{2 n} \longrightarrow 0 .
$$

The homology of this complex is $\operatorname{Tor}^{R}(M, Q)$. By (2.4), $p d_{R} M<\infty \Leftrightarrow(2.6)$ is acyclic, and, clearly, a necessary and sufficient condition for (2.6) to be acyclic is that $\operatorname{rank} A+\operatorname{rank} A^{\vee}=2 n$. Q.E.D.

(2.7) Remarks. There is an involutive $K$-automorphism $\alpha$ of $R$ such that $\alpha$ maps $x_{1}, x_{2}, x_{3}, x_{4}$ to $x_{4},-x_{2},-x_{3}, x_{1}$ respectively. Consider the map $R \stackrel{\alpha}{\longrightarrow} R$. Both restriction of scalars and extension of scalars give the same exact functor, which we shall denote ${ }^{\vee}$, from $R$-modules to themselves. (To see this let us write $R=R_{0} \stackrel{\alpha}{\longrightarrow} R \stackrel{\alpha}{\longrightarrow} R_{2}=R$. Then there is a natural isomorphism $R_{2} \otimes_{R} M \cong{ }_{R_{0}} M$ if we identify both $R_{2}$ and $R_{0}$ as $R$ again: in fact, the map is given by $r_{2} \otimes u \mapsto \alpha\left(r_{2}\right) u$.)

Evidently, if $M$ has finite length $n$ and has $2 n \times 2 n$ matrix $A$, the matrix of $M^{\vee}$ is $A^{\vee}$.

We also note that the Matlis dual $M^{*}$ of $M$, which may be thought of as $\operatorname{Hom}_{K}(M, K)$, has $2 n \times 2 n$ matrix

$$
A^{*}=\left[\begin{array}{ll}
A_{1}^{t} & A_{2}^{t} \\
A_{3}^{t} & A_{4}^{t}
\end{array}\right], \quad \text { where " } t \text { " denotes transpose. }
$$
This matrix has the same rank as its transpose $\left[\begin{array}{ll}A_{1} & A_{3} \\ A_{2} & A_{4}\end{array}\right]$ (they are, of
course, similar as $2 n \times 2 n$ matrices), and

$$
\left[\begin{array}{rr}
0 & 1 \\
-1 & 0
\end{array}\right]\left[\begin{array}{ll}
A_{1} & A_{3} \\
A_{2} & A_{4}
\end{array}\right]\left[\begin{array}{rr}
0 & -1 \\
0 & 0
\end{array}\right]=\left[\begin{array}{rr}
A_{4} & -A_{2} \\
-A_{3} & A_{1}
\end{array}\right]
$$

so that $A^{*}$ and $A^{\vee}$ are similar as $2 n \times 2 n$ matrices and have the same rank. 
(2.8) Proposition. Let $M$ be any R-module of finite length $n$ and let $A$ be the corresponding $2 n \times 2 n$ matrix. Then

and

$$
l\left(Q \otimes_{R} M\right)=l\left(P \otimes_{R} M^{*}\right)=l\left(P \otimes_{R} M^{\vee}\right)=2 n-\operatorname{rank} A
$$

$$
l\left(P \otimes_{R} M\right)=l\left(Q \otimes_{R} M^{*}\right)=l\left(Q \otimes_{R} M^{\vee}\right)=2 n-\operatorname{rank} A^{\vee} .
$$

Proof. Since $R^{2} \stackrel{X}{\rightarrow} R^{2} \rightarrow Q \rightarrow 0$ is a presentation of $Q$, if we apply $\otimes_{R} M$ we find $Q \otimes_{R} M \cong \operatorname{Coker} A$, which implies $l\left(Q \otimes_{R} M\right)=2 n-\operatorname{rank} A$. Similarly $l\left(P \otimes_{R} M\right)=2 n-\operatorname{rank} A^{\vee}$. Replacing $M$ by $M^{\vee}$ interchanges $A$ and $A^{\vee}$ here. Replacing $M$ by $M^{*}$ results in the substitution of $A^{*}$ for $A$ : but $A^{*}$ has the same rank as $A^{\vee}$ and $A^{* \vee}=A^{\vee *}$ has the same rank as $A^{\vee \vee}=A$. Q.E.D.

(2.9) Proposition. Let $M$ be an $R$-module of finite length $n$ and finite projective dimension, and let $A$ be the corresponding matrix. Then

and

$$
\chi(M, R / Q)=\chi\left(M^{\vee}, R / P\right)=\chi\left(M^{*}, R / P\right)=\operatorname{rank} A-n
$$

$$
\chi(M, R / P)=\chi\left(M^{\vee}, R / Q\right)=\chi\left(M^{*}, R / Q\right)=\operatorname{rank} A^{\vee}-n .
$$

(Note: $\operatorname{rank} A^{\vee}-n=n-\operatorname{rank} A=-(\operatorname{rank} A-n)$ here.)

Proof. $\chi(M, R / Q)=\chi(M, R)-\chi(M, Q)=l(M \otimes R)-l(M \otimes Q)$, since $R$ and $Q$ are Cohen-Macaulay of dimension 3 , and this is $l(M)-l(M \otimes Q)=n-(2 n-\operatorname{rank} A)$ $=\operatorname{rank} A-n$. The other parts follows similarly, using (2.8). Q.E.D.

\section{3. $R$-modules killed by $\mathrm{m}^{3}+\left(x_{2}, x_{4}\right) \mathrm{m}$}

The objective of this section is a detailed study of finite length $R$-modules killed by $\mathrm{m}^{3}+\left(x_{2}, x_{4}\right) \mathrm{m}$. Ultimately we shall construct such modules $M$ with $p d_{R} M$ finite and $\chi_{R}(M, R / Q)<0$.

First consider an arbitrary finite length $R$-module $M$ with $l(M)=n$ such that $\mathrm{m}^{3} M=0$. Choose a filtration

$$
M=M_{0} \supset M_{1} \supset M_{2} \supset M_{3}=0
$$

such that $m_{i} \subset M_{i+1}, 0 \leqq i \leqq 2$. E.g. we may choose $M_{i}=\mathfrak{m}^{i} M, 0 \leqq i \leqq 3$, or $M_{i}$ $=\mathrm{Ann}_{M} \mathrm{~m}^{3-i}, 0 \leqq i \leqq 3$ : if these two filtrations are different, many choices may be possible. Let $t, s, r$ denote the respective lengths of $M / M_{1}, M_{1} / M_{2}$, and $M_{2}$, and let $u_{1}, \ldots, u_{r}, u_{r+1}, \ldots, u_{r+s}$, and $u_{r+s+1}, \ldots, u_{r+s+1}$ be sets of vectors in $M_{2}, M_{1}$, and $M$ respectively whose images in the respective quotient vector spaces $M_{2} / M_{3}=M_{2}, M_{1} / M_{2}$, and $M / M_{1}$ are $K$-vector space bases for those quotients. Thus, $u_{1}, \ldots, u_{n}$ is a $K$-basis for $M$. Let $U^{r}, V^{s}$, and $W^{t}$ denote $\sum_{i=1}^{r} K u_{i}, \sum_{i=r+1}^{r+s} K u_{i}$, and $\sum_{i=r+s+1}^{r+s+t} K u_{i}$, respectively.

Multiplication by $x_{i}$ maps $W^{t} \rightarrow U^{r} \oplus V^{s} \cong U^{r} \times V^{s}$, maps $V^{s} \rightarrow U^{r}$, and kills $U^{r}$, and so is determined by the matrices $a_{i}, b_{i}, c_{i}$ of the induced maps

$$
V^{s} \rightarrow U^{r}, \quad W^{t} \rightarrow V^{s}, \quad W^{t} \rightarrow U^{r}
$$


respectively. In block form the matrix $A_{i}$ of multiplication by $x_{i}$ with respect to the basis $u_{1}, \ldots, u_{n}$ is

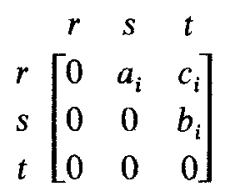

where the letters " $r$ ", " $s$ ", " $t$ " have been placed to indicate the sizes of the blocks. For matrices $A_{i}$ with this block form we have $A_{i} A_{j}=\left[\begin{array}{ccc}0 & 0 & a_{i} b_{i} \\ 0 & 0 & 0 \\ 0 & 0 & 0\end{array}\right]$, so that $A_{i} A_{j}=A_{j} A_{i}$ if and only if $a_{i} b_{j}=a_{j} b_{i}$, while $A_{i} A_{j} A_{k}=0$ automatically for all choices of $i, j, k$.

Thus, any four such matrices $A_{i}$ in block form satisfying $a_{i} b_{j}=a_{j} b_{i}$ for all $i, j$ and $a_{1} b_{4}=a_{2} b_{3}$ determine an $R$-module $M$ having a filtration in which the lengths of the factors are $r, s, t$.

Now suppose, in addition, that $\left(x_{2}, x_{4}\right) \mathfrak{m} M=0$, or, equivalently, that $\left(x_{2}, x_{4}\right) M \subset$ Ann $_{M} m$. Then we may choose a filtration which satisfies the additional conditions $\left(x_{2}, x_{4}\right) M \subset M_{2}$ and $\left(x_{2}, x_{4}\right) M_{1}=0$, e.g. $M_{1}=\mathrm{m} M, M_{2}$ $=\mathfrak{m}^{2} M+\left(x_{2}, x_{4}\right) M\left(\subset \mathfrak{m} M \cap \mathrm{Ann}_{M} \mathfrak{m}\right)$.

These constraints on the filtration imply that $a_{2}=a_{4}=0$ and $b_{2}=b_{4}=0$. The $A_{i}$ then have the following form:

$$
\begin{array}{rlrl}
A_{1} & =\left[\begin{array}{ccc}
0 & a_{1} & c_{1} \\
0 & 0 & b_{1} \\
0 & 0 & 0
\end{array}\right] & A_{2}=\left[\begin{array}{ccc}
0 & 0 & c_{2} \\
0 & 0 & 0 \\
0 & 0 & 0
\end{array}\right] \\
A_{3}=\left[\begin{array}{ccc}
0 & a_{3} & c_{3} \\
0 & 0 & b_{3} \\
0 & 0 & 0
\end{array}\right] & A_{4}=\left[\begin{array}{llc}
0 & 0 & c_{4} \\
0 & 0 & 0 \\
0 & 0 & 0
\end{array}\right]
\end{array}
$$

We also note:

(3.2) Given matrices $A_{i}$ as in (3.1) above:

a) $A_{i} A_{j} A_{k}=0$ for all $i, j, k$.

b) $A_{i} A_{j}=A_{j} A_{i}=0$ if $i$ or $j$ is even.

c) The $A_{i}$ commute in pairs if and only if $A_{1} A_{3}=A_{3} A_{1}$, and this holds if and only if $a_{1} b_{3}=a_{3} b_{1}$.

Thus, to give an $R$-module $M$ of length $n$ killed by $\mathrm{m}^{3}+\left(x_{2}, x_{4}\right) \mathrm{m}$ is equivalent to giving nonnegative integers $r, s, t$ with $r+s+t=n$ together with two $r \times s$ matrices $a_{1}, a_{3}$ over $K$, two $s \times t$ matrices $b_{1}, b_{3}$ over $K$ satisfying

$$
a_{1} b_{3}=a_{3} b_{1}
$$

and four arbitrary $s \times t$ matrices $c_{i}$ over $K$. The integers $r, s, t$ and the matrices $a_{i}, b_{i}, c_{i}$ are not uniquely determined by $M$, but every $M$ arises from data of this sort. 
Our next objective is to give necessary and sufficient conditions on $a_{1}, a_{3}, b_{1}, b_{3}$ satisfying (3.3) above for there to exist $c_{i}$ such that the corresponding module $M$ has finite projective dimension. We first note

(3.4) Lemma. Let $q, r, s, t>0$ be integers. Let $a, b$ be fixed $q \times s$ and $r \times t$ matrices, respectively, over a field $K$. Let $\operatorname{rank} a=\alpha$ and $\operatorname{rank} b=\beta$. Let $\mathscr{M}_{q, \mathrm{t}}$ be the variety of $q \times t$ matrices over $K$ (of course $\mathscr{M}_{q, t}$ may be identified with $\mathbf{A}_{\mathbf{K}}^{q t}$ ). Then the maximum possible rank for a matrix whose block form is

$$
\begin{gathered}
s \\
r \\
r \\
{\left[\begin{array}{ll}
a & c \\
0 & b
\end{array}\right]}
\end{gathered}
$$

as $c$ varies in $\mathscr{M}_{q, t}$ is $\min \{q+\beta, t+\alpha\}$, and this maximum is achieved on a nonempty Zariski open subset of $\mathscr{M}_{q, t}$ (consequently dense when $K$ is infinite, but nonempty even when $K$ is finite).

Proof. We may perform column operations on the first $s$ columns and row operations on the last $r$ rows without affecting the issue. We may also perform row operations on the first $q$ rows and column operations on the last $t$ columns: the only observation we need is that the effect on $c$ is simply an invertible linear change of coordinates in $\mathscr{M}_{q, t} \cong \mathbf{A}_{K}^{q t}$. Hence there is no loss of generality in assuming

$$
a=\left[\begin{array}{cc}
1_{\alpha} & 0 \\
0 & 0
\end{array}\right], \quad b=\left[\begin{array}{cc}
1_{\beta} & 0 \\
0 & 0
\end{array}\right], \quad \text { and then }\left[\begin{array}{ll}
a & c \\
0 & b
\end{array}\right]=\left[\begin{array}{cccc}
1_{\alpha} & 0 & c_{1} & c_{2} \\
0 & 0 & c_{3} & c_{4} \\
0 & 0 & 1_{\beta} & 0 \\
0 & 0 & 0 & 0
\end{array}\right],
$$

and the rank of this last matrix is easily seen to be $\alpha+\beta+\operatorname{rank} c_{4}$. Since $c_{4}$ is size $(q-\alpha) \times(t-\beta)$, its maximum possible rank is $\min \{q-\alpha, t-\beta\}$, and so the maximum possible $\operatorname{rank}$ for $A$ is $\alpha+\beta+\{\min q-\alpha, t-\beta\}=\min \{q+\beta, t$ $+\alpha\}$. Q.E.D.

(3.5) Notation. Given a pair of matrices $a_{1}, a_{3}$ (respectively, $b_{1}, b_{3}$ ) of the same size over a field $K$ we let $\alpha_{h}$ (respectively, $\beta_{h}$ ) denote the rank of $\left[a_{1} a_{3}\right]$ (respectively $\left[b_{1} b_{3}\right]$ ) and $\alpha_{v}$ (respectively, $\beta_{v}$ ) the rank of $\left[\begin{array}{l}a_{1} \\ a_{3}\end{array}\right]$ (respectively, $\left.\left[\begin{array}{l}b_{1} \\ b_{3}\end{array}\right]\right)$. (The subscripts " $h$ " and " $v$ " stand for "horizontal" and "vertical", respectively.)

We shall see later that the modules $M$ over $R$ of finite length and finite projective dimension such that $m^{2} M=0$ behave as expected with respect to multiplicities. Hence, our real interest is in the case $r, s, t>0$.

(3.6) Proposition. Let $r, s, t>0$ be given integers and let $a_{1}, a_{3}$ be $r \times s$ and $b_{1}, b_{3}$ be $s \times t$ matrices over an infinite field $K$ such that $a_{1} b_{3}=a_{3} b_{1}$.

Then there exist $r \times t$ matrices $c_{i}, 1 \leqq i \leqq 4$, such that the matrix $A$ determined from the $a_{i}, b_{i}, c_{i}$ as in (3.1) corresponds to a module $M$ of finite projective dimension if and only if the following three conditions hold: 
a) $a_{v}=\beta_{h}=s$.

b) $\alpha_{h} \geqq s+2(r-t)$.

c) $\beta_{v} \geqq s-2(r-t)$.

If these conditions hold then $\operatorname{rank} A=2 t+s, \operatorname{rank} A^{\vee}=2 r+s$, and, hence, $\operatorname{rank} A=\operatorname{rank} A^{\vee}$ if and only if $t=r$.

Proof. Let $n=r+s+t$. By Theorem (2.5), $p d_{R} M<\infty \Leftrightarrow \operatorname{rank} A+\operatorname{rank} A^{\vee}=2 n$ $=2(r+s+t)$. Now

$$
A=\left[\begin{array}{cccccc}
0 & a_{1} & c_{1} & 0 & 0 & c_{2} \\
0 & 0 & b_{1} & 0 & 0 & 0 \\
0 & 0 & 0 & 0 & 0 & 0 \\
0 & a_{3} & c_{3} & 0 & 0 & c_{4} \\
0 & 0 & b_{3} & 0 & 0 & 0 \\
0 & 0 & 0 & 0 & 0 & 0
\end{array}\right]
$$

After dropping the rows and columns which are 0 and permuting the rows we obtain:

$$
2 s\left[\begin{array}{c|cc}
s & \multicolumn{2}{c}{2 t} \\
2 r & c_{1} & c_{2} \\
a_{3} & c_{3} & c_{4} \\
\hline 0 & b_{1} & 0 \\
0 & b_{3} & 0
\end{array}\right] .
$$

By Lemma (3.4) for any choice of the $c_{i}$ in sufficiently general position (i.e. off a proper closed subvariety of $\left.\mathscr{M}_{2 r, 2 t} \cong \mathbf{A}_{K}^{4 r t}\right)$ the rank of this matrix will be $\min \left\{2 r+\beta_{v}, 2 t+\alpha_{v}\right\}$. Similarly,

$$
A^{\vee}=\left[\begin{array}{cccccc}
0 & 0 & c_{4} & 0 & 0 & -c_{2} \\
0 & 0 & 0 & 0 & 0 & 0 \\
0 & 0 & 0 & 0 & 0 & 0 \\
0 & -a_{3} & -c_{3} & 0 & a_{1} & c_{1} \\
0 & 0 & -b_{3} & 0 & 0 & b_{1} \\
0 & 0 & 0 & 0 & 0 & 0
\end{array}\right]
$$

and after dropping the rows and columns which are 0 and permuting the rows and columns we get:

$$
2 r\left[\begin{array}{rr|rr}
0 & 0 & c_{4} & -c_{2} \\
-a_{3} & -a_{1} & -c_{3} & c_{1} \\
\hline 0 & 0 & -b_{3} & b_{1}
\end{array}\right]
$$

Since $\beta_{h}=\operatorname{rank}\left[b_{1} b_{3}\right]=\operatorname{rank}\left[-b_{3} b_{1}\right]$ while

$$
\alpha_{h}=\operatorname{rank}\left[a_{1} a_{3}\right]=\operatorname{rank}\left[-a_{3} a_{1}\right]=\operatorname{rank}\left[\begin{array}{cc}
0 & 0 \\
-a_{3} & a_{1}
\end{array}\right],
$$


we again obtain from Lemma (3.4) that for $c_{i}$ in sufficiently general position we have rank $A^{v}=\min \left\{2 r+\beta_{h}, 2 t+\alpha_{h}\right\}$.

The sum of the min's is the same as the min of the four possible "cross sums", and the condition for $p d_{R} M<\infty$ becomes:
i) $2 r+\beta_{v}+2 r+\beta_{h} \geqq 2 r+2 s+2 t$ or
i') $\beta_{v}+\beta_{h} \geqq 2 s-2(r-t)$
ii) $2 r+\beta_{v}+2 t+\alpha_{h} \geqq 2 r+2 s+2 t$ or
ii') $\beta_{v}+\alpha_{h} \geqq 2 s$
iii) $2 t+\alpha_{v}+2 r+\beta_{h} \geqq 2 r+2 s+2 t$ or
iii') $\beta_{h}+\alpha_{v} \geqq 2 s$
iv) $2 t+\alpha_{v}+2 t+\alpha_{h} \geqq 2 r+2 s+2 s$ or
iv') $\alpha_{v}+\alpha_{h} \geqq 2 s+2(r-t)$

(These conditions say that $\operatorname{rank} A+\operatorname{rank} A^{\vee} \geqq 2 n$ : of course, when this happens we actually get equality.) Now, $\beta_{h} \leqq s$ since $\left[b_{1} b_{3}\right]$ is $s \times 2 t$ and $\alpha_{v} \leqq s$ since $\left[\begin{array}{l}a_{1} \\ a_{3}\end{array}\right]$ is $2 r \times s$. Thus, iii') holds if and only if $\beta_{h}=\alpha_{v}=s$. If we substitute $s$ for $\beta_{h}, \alpha_{v}$ in $\mathrm{i}^{\prime}$ ), iv') and subtract $s$ from both sides we obtain the conditions b), c) stated in the proposition, while ii') is redundant: it follows from b) and c) by adding them.

By taking the $c_{i}$ in general position we can simultaneously maximize the ranks of $A$ and $A^{v}$. But then $\operatorname{rank} A=\min \left\{2 r+\beta_{v}, 2 t+s\right\}=2 t+s$ (by b)) and $\operatorname{rank} A^{\vee}=\min \left\{2 r+s, 2 t+\alpha_{h}\right\}=2 r+s($ by c)). Q.E.D.

(3.7) Theorem. Let $K$ be an infinite field. Let $r, s, t>0$ be integers. Let $a_{1}, a_{3}$ be given $r \times s$ matrices over $K$.

Let $\delta=\delta\left(a_{1}, a_{3}\right)$ be the greatest integer such that there exist $\delta$-dimensional subspaces $D_{1}, D_{3} \subset K^{s}$ and an isomorphism $\lambda: D_{1} \cong D_{3}$ satisfying $D_{1} \cap D_{3}=0$ and $a_{1} \lambda=\left.a_{3}\right|_{D_{1}}$ (i.e. $\delta$ is the greatest integer such that there exist vectors $d_{11}, \ldots, d_{1 \delta}$, $d_{31}, \ldots, d_{3 \delta}$ in $K^{s}$ all independent, such that $a_{1}\left(d_{3 i}\right)=a_{3}\left(d_{1 i}\right), 1 \leqq i \leqq \delta$ : $d_{11}, \ldots, d_{1 \delta}$ correspond to a basis for $D_{1}$, and $d_{31}, \ldots, d_{3 \delta}$ to its image under $\left.\lambda\right)$.

Let $E=E\left(a_{1}, a_{3}\right)=a_{1}^{-1}\left(\operatorname{Im} a_{1} \cap \operatorname{Im} a_{3}\right)+a_{3}^{-1}\left(\operatorname{Im} a_{1} \cap \operatorname{Im} a_{3}\right)$.

Let $Z$ be the vector space consisting of all $2 s \times 1$ column vectors $\left[\begin{array}{l}z_{1} \\ z_{3}\end{array}\right]$ with $z_{1}, z_{3} \in K^{s}$ such that $a_{1} z_{3}=a_{3} z_{1}$.

Then $\operatorname{dim}_{K} Z=2 s-\alpha_{h}$ (see (3.5) for notation).

Moreover, there exist $s \times t$ matrices $b_{1}, b_{3}$ over $K$ satisfying

a) $a_{1} b_{3}=a_{3} b_{1}$ and

b) 1) $\alpha_{v}=\beta_{h}=s$,

2) $\alpha_{h} \geqq s+2(r-t)$, and

3) $\beta_{v} \geqq s-2(r-t)$

if and only if the following conditions hold:

c) 1) $\operatorname{Ker} a_{1} \cap \operatorname{Ker} a_{3}=0$,

2) $E=K^{s}$

3) $\alpha_{h}-s$ is even, and $t=r-\frac{\alpha_{h}-s}{2}$,

4) $2 r+2 \delta \geqq \alpha_{h}+s$, and

5) $2 r+\alpha_{h} \geqq 3 s$.

If these conditions hold $b_{1}, b_{3}$ may be chosen by taking $\left[\begin{array}{l}b_{1} \\ b_{3}\end{array}\right]$ to consist of $t$ columns in sufficiently general position in $Z$. 
The modules $M$ of finite length $n=r+s+t$ and finite projective dimension which may then be constructed by further choosing $c_{i}$ in sufficiently general position as in Proposition (3.6) will satisfy:

$$
\begin{aligned}
& \chi_{R}(M, R / P)=r-t=\frac{\alpha_{h}-s}{2} \\
& \chi_{R}(M, R / Q)=t-r=-\frac{\alpha_{h}-s}{2} .
\end{aligned}
$$

Proof. First note that there is a surjection $Z \rightarrow \operatorname{Im} a_{1} \cap \operatorname{Im} a_{3}$ which maps $\left[\begin{array}{l}b_{1} \\ b_{3}\end{array}\right] \mapsto a_{3}\left(b_{1}\right)\left(=a_{1}\left(b_{3}\right)\right)$. The kernel consists of precisely those columns such that $b_{1} \in \operatorname{Ker} a_{3}$ and $b_{3} \in \operatorname{Ker} a_{1}$ and so is isomorphic to $\operatorname{Ker} a_{1} \times \operatorname{Ker} a_{3}$.

Also, $Z$ may be identified with the kernel of $\left[a_{3}-a_{1}\right]$ so that $\operatorname{dim}_{K} Z=2 \mathrm{~s}$ $-\operatorname{rank}\left[a_{3}-a_{1}\right]$ and $\operatorname{rank}\left[a_{3}-a_{1}\right]=\operatorname{rank}\left[a_{1} a_{3}\right]=\alpha_{h}$.

Now, it is clear that $\beta_{h} \leqq s$, and $\beta_{h}, \beta_{v}$ will be maximized when the $t$ columns of $\left[\begin{array}{l}b_{1} \\ b_{3}\end{array}\right]$ are chosen in general position in $Z$. When this is done it is easy to see that

$$
\beta_{h}= \begin{cases}2 t & \text { if } t \leqq \delta \\ \delta+t & \text { if } \delta \leqq t \leqq \operatorname{dim}_{K} E-\delta \\ \operatorname{dim}_{K} E & \text { if } t \geqq \operatorname{dim}_{K} E .\end{cases}
$$

To verify this, first note that, by the definition of $\delta$, for $t \leqq \delta$ we can choose $\left[\begin{array}{l}b_{1} \\ b_{3}\end{array}\right]=\left[\begin{array}{l}d_{11} \ldots d_{1 t} \\ d_{31} \ldots d_{3 t}\end{array}\right]$ where $d_{11}, \ldots, d_{1 t}, d_{31}, \ldots, d_{3 t}$ are all independent. Also note that for all admissible choices of $b_{1}, b_{3}, \operatorname{Im} b_{i} \subset \operatorname{Im} b_{1} \cap \operatorname{Im} b_{3}, i=1,3$, so that $\operatorname{Im}\left[b_{1} b_{3}\right] \subset E$. As we choose additional columns for $\delta \leqq t \leqq \operatorname{dim}_{K} E-\delta$, so long as the span of all columns chosen so far in $\left[b_{1} b_{3}\right]$ is not all of $E$ we can choose a new column so that at least one of the two vectors which occur is not in the span of those already chosen. For $t$ in this range the rank of $\left[b_{1} b_{3}\right]$ will be $2 \delta+(t-\delta)=\delta+t$. When $t=\operatorname{dim}_{K} E-\delta$ this is $\operatorname{dim}_{K} E$, at which point $\operatorname{Im}\left[b_{1} b_{3}\right]=E$ and cannot grow further.

It is worth noting that $2 \delta \leqq \operatorname{dim}_{K} E \leqq s$.

Thus, in order that $\beta_{h}=s$ we must have $\operatorname{dim}_{K} E=s$ and $t \geqq \operatorname{dim}_{K} E-\delta=s-\delta$.

Moreover, with the columns of $\left[\begin{array}{l}b_{1} \\ b_{3}\end{array}\right]$ in general position we have

$$
\beta_{v}= \begin{cases}t & \text { if } t \leqq 2 s-\alpha_{h} \\ 2 s-\beta_{h} & \text { if } t \geqq 2 s-\alpha_{h}\end{cases}
$$

since $\operatorname{dim}_{K} Z=2 s-\alpha_{h}$.

Now, condition a) is simply equivalent to requiring that the columns of $\left[\begin{array}{l}b_{1} \\ b_{3}\end{array}\right]$ lie in $Z$ while condition b), i.e.

1) $\alpha_{v}=\beta_{h}=s$

2) $\alpha_{h} \geqq s+2(r-t)$ or $\left.2^{\prime}\right) \alpha_{h}-s \geqq 2(r-t)$

3) $\beta_{v} \geqq s-2(r-t)$ 
may now be analyzed as follows:

$\alpha_{v}=s$ is easily seen equivalent to $c 1$ ), $\operatorname{Ker} a_{1} \cap \operatorname{Ker} a_{3}=0$, and, as we have already seen, for the case where the columns of the matrix $\left[\begin{array}{l}b_{1} \\ b_{3}\end{array}\right]$ are chosen in general position in $Z, \beta_{h}=s$ is equivalent to $\operatorname{dim}_{K} E=s$ and $t \geqq \operatorname{dim}_{K} E-\delta=s$ $-\delta$. The condition $\operatorname{dim}_{K} E=s$ is evidently equivalent to $E=K^{s}$. This explains conditions $\mathrm{c} 1$ ) and $\mathrm{c} 2$ ). From our remarks above, with the columns of $\left[\begin{array}{l}b_{1} \\ b_{3}\end{array}\right]$ in general position in $Z$ (which gives the best chance of satisfying b1), b3)) we have $\beta_{v}=\min \left\{t, 2 s-\alpha_{h}\right\}$ and 3) becomes

and

$$
\left.t \geqq s-2(r-t) \text { or } 3^{\prime}\right) \quad t \leqq 2 r-s
$$

$$
\left.2 s-\alpha_{h} \geqq s-2(r-t) \text { or } 3^{\prime \prime}\right) 2(r-t) \geqq \alpha_{h}-s .
$$

Clearly, $\left.2^{\prime}\right)$ and $\left.3^{\prime \prime}\right)$ together are simply equivalent to $2(r-t)=\alpha_{h}-s$ or $t=r$ $-\frac{\alpha_{h}-s}{2}$, which gives $\mathrm{c} 3$ ). Hence all conditions are satisfied if and only if the two remaining inequalities:

$$
\left.t \geqq s-\delta \quad \text { and } \quad 3^{\prime}\right) t \leqq 2 r-s
$$

are satisfied. Since $t=r-\frac{\alpha_{h}-s}{2}$ the first becomes $r-\frac{\alpha_{h}-s}{2} \geqq s-\delta$ or $2 r-\alpha_{h}$ $+s \geqq 2 s-2 \delta$ or $2 r+2 \delta \geqq \alpha_{h}+s$, which is c4), while $3^{\prime}$ ) becomes $r-\frac{\alpha_{h}-s}{2} \leqq 2 r$ $-s$ or $2 r-\alpha_{h}+s \leqq 4 r-2 s$ or $2 r+\alpha_{h} \geqq 3 s$ which is $\mathrm{c} 5$ ).

It remains only to prove the last assertion. By Proposition (2.9) $\chi_{R}(M, R / P)$ will be rank $A^{\vee}-n=$ (by the last part of Proposition (3.6)) $2 r+s-(r+s+t)=r$ $-t=\frac{\alpha_{h}-s}{2}$, by $\left.\mathrm{c} 3\right)$. The argument for $\chi_{R}(M, R / Q)$ is similar. Q.E.D.

(3.8) Remarks. Certain notions which we have discussed in matrix terms can be viewed more "invariantly" in module-theoretic terms.

For example, the maps $a_{i}$ were introduced as maps $V^{s} \rightarrow U^{r}$. But $V^{s} \cong M_{1} / M_{2}$ and $U^{r}=M_{2}$. Since each $x_{i}$ kills $M_{2}$, there is an induced map $x_{i}: M_{1} / M_{2} \rightarrow M_{2}$. In these terms, $\alpha_{h}=\operatorname{rank}\left[a_{1} a_{3}\right]=\operatorname{dim}_{K}\left(\operatorname{Im} a_{1}+\operatorname{Im} a_{3}\right)$ $=l\left(\left(x_{1}, x_{3}\right) M_{1}\right)$.

Likewise, although $b_{i}$ was introduced as a map $W^{t} \rightarrow V^{s}, W^{t} \cong M / M_{1}$, and $b_{i}$ simply corresponds to the map $M / M_{1} \rightarrow M_{1} / M_{2}$ induced by multiplication by $x_{i}$. We shall leave most other similar reinterpretations to the reader.

\section{The main results}

In this section we study in detail pairs of matrices $a_{1}, a_{3}$, of the same size, which satisfy the conditions $c 1$ )-c5) listed in (3.7). We call such a pair admissible. 
By Theorem (3.7), the problem of finding modules $M$ of finite length and finite projective dimension over $R$ such that $\mathrm{m}^{3}+\left(x_{2}, x_{4}\right) \mathrm{m}$ kills $M$ and $\chi(M, R / P) \neq 0$ is equivalent to finding pairs of matrices $a_{1}, a_{3}$ over $K$ of the same size, say $r \times s$, which are admissible, and such that $\alpha_{h} \neq s$.

Henceforth, we assume that $K$ is an infinite field. $a_{1}, a_{3}$ will always denote matrices of the same size. We write $r\left(a_{1}\right)$ or $r\left(a_{3}\right)$ for the number of rows, and $s\left(a_{1}\right)$ or $s\left(a_{3}\right)$ for the number of columns.

We write $\alpha\left(a_{1}, a_{3}\right)$ for $\operatorname{rank}\left[a_{1} a_{3}\right]=\operatorname{dim}_{K}\left(\operatorname{Im} a_{1}+\operatorname{Im} a_{3}\right)$ : this is a slight change from the preceding section, where the notation " $\alpha_{h}$ " was used. We may omit the variable matrices $a_{1}, a_{3}$ from the notation if the meaning is clear. We define $t\left(a_{1}, a_{3}\right)=r-\frac{\alpha-s}{2}$ and $n\left(a_{1}, a_{3}\right)=r+s+t$.

$\delta\left(a_{1}, a_{3}\right)$ has the same meaning as in the second paragraph of Theorem

Let $G\left(a_{1}, a_{3}\right)=\operatorname{Im} a_{1} \cap \operatorname{Im} a_{3}$ and $H=a_{1}^{-1}(G) \cap a_{3}^{-1}(G)$.

Let $g\left(a_{1}, a_{3}\right), h\left(a_{1}, a_{3}\right)$ denote the dimensions of $G$ and $H$, respectively.

Let $h_{i}^{\prime}\left(a_{1}, a_{3}\right)=\operatorname{dim} a_{i}^{-1}(G)-h$, a nonnegative integer.

The following result is the main technical tool we need in our analysis of the desired modules $M$.

(4.1) Theorem. Let $K$ be an infinite field. Let $g, h$ be fixed nonnegative integers. Then there exists an admissible pair of matrices $a_{1}, a_{3}$ over $K$ of some size $r \times s$ such that $g\left(a_{1}, a_{3}\right)=g$ and $h\left(a_{1}, a_{3}\right)=h$ if and only if :

1) $g-h$ is even, and

2) $h \leqq 2 g$.

For fixed $g, h$ satisfying 1) and 2) above and given integers $h_{1}^{\prime}, h_{3}^{\prime}$ one can construct $a_{1}, a_{3}$ such that $\mathrm{g}\left(a_{1}, a_{3}\right)=g, h\left(a_{1}, a_{3}\right)=h$, and $h_{i}^{\prime}\left(a_{1}, a_{3}\right)=h_{i}^{\prime}, i=1,3$, if and only if

3) $h_{1}^{\prime}, h_{3}^{\prime} \geqq g-h$

where $g-h=\max \{g-h, 0\}$. For such $a_{1}, a_{3}$ :

4) $s=h+h_{1}^{\prime}+h_{3}^{\prime}$

5) $\alpha=g+h_{1}^{\prime}+h_{3}^{\prime}$ and, hence,

6) $\frac{\alpha-s}{2}=\frac{g-h}{2}$ and

7) $t=r-\frac{g-h}{2}$. Also,

8) $n=h+h_{1}^{\prime}+h_{3}^{\prime}-\frac{g-h}{2}+2 r$.

One can choose $a_{1}, a_{3}$ so that $\delta \geqq(h-g) / 2$ and the smallest values one can take for $r, n$ for fixed $g, h, h_{1}^{\prime}, h_{3}^{\prime}$ satisfying 1), 2), 3) are

9) $r=h_{1}^{\prime}+h_{3}^{\prime}+\max \left\{g, \frac{3}{2} h-\frac{1}{2} g\right\}$

10) $n=3\left(h+h_{1}^{\prime}+h_{3}^{\prime}\right)+\frac{3}{2}|g-h|=3 s+\frac{3}{2}|g-h|$. 
For fixed $g, h$ satisfying 1) and 2) the smallest possible values for $r, n$ are achieved when $h_{1}^{\prime}=h_{3}^{\prime}=\mathrm{g}-h$.

Moreover, if a module $M$ of finite projective dimension over $R$ and length $n$ is constructed from $a_{1}, a_{3}$ as in Theorem (3.7), then

$$
\chi(M, R / P)=\frac{g-h}{2} \text { and } \chi(M, R / Q)=\frac{h-g}{2} .
$$

Proof. First consider $r \times s$ matrices $a_{1}, a_{3}$ satisfying conditions $\left.\left.\mathrm{c} 1\right)-\mathrm{c} 5\right)$ of Theorem (3.7). Since $a_{1}^{-1}(G)+a_{2}^{-1}(G)=K^{s}$, one can think of $H_{i}=a_{i}^{-1}(G)$ as $H \oplus H_{i}^{\prime}$, where $\operatorname{dim} H_{i}^{\prime}=h_{i}^{\prime}$ and so $s=h+h_{1}^{\prime}+h_{3}^{\prime}$ as asserted in 4): in fact $K^{s}$ $=H \oplus H_{1}^{\prime} \oplus H_{3}^{\prime}$. Now,

$$
\begin{aligned}
\operatorname{Im} a_{1}+\operatorname{Im} a_{3} & =a_{1}\left(H_{1} \oplus H_{3}^{\prime}\right)+a_{3}\left(H_{3} \oplus H_{1}^{\prime}\right) \\
& =G+a_{1}\left(H_{3}^{\prime}\right)+G+a_{3}\left(H_{1}^{\prime}\right)=G+a_{1}\left(H_{3}^{\prime}\right)+a_{3}\left(H_{1}^{\prime}\right) .
\end{aligned}
$$

But, $\operatorname{Ker} a_{1} \cap H_{3}^{\prime}=0$, for

$$
\operatorname{Ker} a_{1} \cap H_{3}^{\prime} \subset H_{1} \cap H_{3}^{\prime}=H_{1} \cap\left(H_{3} \cap H_{3}^{\prime}\right)=\left(H_{1} \cap H_{3}\right) \cap H_{3}^{\prime}=H \cap H_{3}^{\prime}=0,
$$

and, similarly, $H_{1}^{\prime} \cap \operatorname{Ker} a_{3}=0$. Thus, $a_{i}\left(H_{4-i}^{\prime}\right) \cong H_{4-i}^{\prime}, \quad i=1,3$. Likewise, $G \cap a_{1}\left(H_{3}^{\prime}\right)=0$, for $v \in H_{3}^{\prime}$ and

$$
a_{1}(v) \in G \Rightarrow v \in a_{1}^{-1}(G)=H_{1} \Rightarrow v \in H_{1} \cap H_{3}^{\prime}=0 .
$$

Similarly, $G \cap a_{3}\left(H_{1}^{\prime}\right)=0$, and

$$
a_{1}\left(H_{3}^{\prime}\right) \cap a_{3}\left(H_{1}^{\prime}\right) \subset \operatorname{Im} a_{1} \cap \operatorname{Im} a_{3}=G \Rightarrow a_{1}\left(H_{3}^{\prime}\right) \cap a_{3}\left(H_{1}^{\prime}\right) \subset a_{1}\left(H_{3}^{\prime}\right) \cap G=0 .
$$

Thus, $\operatorname{Im} a_{1}+\operatorname{Im} a_{3} \cong G \oplus a_{1}\left(H_{3}^{\prime}\right) \oplus a_{3}\left(H_{1}^{\prime}\right)=G \oplus H_{3}^{\prime} \oplus H_{1}^{\prime}$ and so $\alpha=g+h_{1}^{\prime}+h_{3}^{\prime}$, as asserted in 5). 6) is immediate from 4) and 5), and 7) (and 8)) from 6) and (3.7c3) (and the definition of $n$ ). Moreover, we have also established condition 1) on $g$ and $h$. Let $K_{i}=\operatorname{Ker} a_{i}$ and $k_{i}=\operatorname{dim}_{K} K_{i}$. Now, $a_{i}$ maps $H_{i}$ onto $G$ and $K_{i} \subset H_{i}$, so that $k_{i}=h+h_{i}^{\prime}-g \geqq 0, i=1,3$, whence $h_{i}^{\prime} \geqq g-h$. Since $h_{i}^{\prime} \geqq 0$ is clear, we have proved 3).

Since $(3.7 \mathrm{c} 1)$ asserts that $K_{1} \cap K_{3}=0$, we must have $k_{1}+k_{3} \leqq s$ or

$$
h+h_{1}^{\prime}-g+h+h_{3}^{\prime}-g \leqq h+h_{1}^{\prime}+h_{3}^{\prime}
$$

which is equivalent to $h \leqq 2 g$. Thus, 2 ) is necessary.

We shall prove the remaining statements in the course of constructing $a_{1}$, $a_{3}$ once $g, h, h_{1}^{\prime}, h_{3}^{\prime}$ have been given.

Now suppose that $g, h, h_{1}^{\prime}, h_{3}^{\prime}$ satisfying 1), 2), and 3) have been specified. We shall show that $a_{1}, a_{3}$ can be constructed for all sufficiently large $r$, and we shall determine the least value of $r$ for which they can be constructed.

Note that we must take $s=h+h_{1}^{\prime}+h_{3}^{\prime}$.

Next, choose arbitrary subspaces $H, H_{1}^{\prime}, H_{3}^{\prime}$ of $K^{s}$ of dimensions $h, h_{1}^{\prime}, h_{3}^{\prime}$ respectively such that $K^{s}=H \oplus H_{1}^{\prime} \oplus H_{3}^{\prime}$. Let $G=K^{g}$ and $T=G \oplus H_{3}^{\prime} \oplus H_{1}^{\prime}$. Then $\operatorname{dim} T=\alpha$ and, of course, we must have $r \geqq \alpha$. We shall construct $a_{1}, a_{3}$ by 
giving maps $K^{s} \rightarrow T$ (which we call $\tilde{a}_{i}$ or, when precision is not needed, $a_{i}$ ) and then composing them with any embedding $T \hookrightarrow K^{r}$ for a suitable $r \geqq \alpha=g+h_{1}^{\prime}$ $+h_{3}^{\prime}$. The earlier part of this proof showed that $a_{1}, a_{3}$ must "arise" in this way, with $T=\operatorname{Im} a_{1}+\operatorname{Im} a_{3}$.

Conditions 1$), 2), 3$ ) of (3.7c) only depend on $\tilde{a}_{1}, \tilde{a}_{3}$, as do $s, \alpha$ and $\delta$. It is then clear that we may choose any value of $r$ such that $r \geqq \alpha, 2 r \geqq \alpha+s-2 \delta$, and $2 r \geqq 3 s-\alpha$, provided that the value of $t=r-\frac{g-h}{2}$ is positive, i.e. such that $r>\frac{g-h}{2}$. Since we already have the condition $r \geqq \alpha=g+h_{1}^{\prime}+h_{3}^{\prime}$, this is automatic. Thus, we may choose any value of $r$ satisfying

$$
2 r \geqq \max \{2 \alpha, \alpha+s-2 \delta, 3 s-\alpha\} .
$$

Clearly, to minimize $r$, we want to construct $\tilde{a}_{1}, \tilde{a}_{3}$ with $\delta$ as large as possible. We shall show that the $\tilde{a}_{i}$ can always be chosen (when 1), 2), 3) hold) with $\delta \geqq \frac{h-g}{2}$. Once we have done this, we need not concern ourselves with whether $\delta$ can be made still larger, for the terms

become

$$
2 \alpha, \quad \alpha+s-2 \delta, \quad 3 s-\alpha
$$

$$
2\left(g+h_{1}^{\prime}+h_{3}^{\prime}\right), \quad g+h_{1}^{\prime}+h_{3}^{\prime}+h+h_{1}^{\prime}+h_{3}^{\prime}-2 \delta, \quad 3\left(h+h_{1}^{\prime}+h_{3}^{\prime}\right)-\left(g+h_{1}^{\prime}+h_{3}^{\prime}\right)
$$

and the second is less than or equal to the first, since $g+h-2 \delta \leqq 2 g \Leftrightarrow \delta$ $\geqq \frac{h-g}{2}$. Thus, the least value of $2 r$ which can be used is $\max \left\{2\left(g+h_{1}^{\prime}+h_{3}^{\prime}\right), 3\left(h+h_{1}^{\prime}+h_{3}^{\prime}\right)-\left(g+h_{1}^{\prime}+h_{3}^{\prime}\right)\right\}=2\left(h_{1}^{\prime}+h_{3}^{\prime}\right)+2\left\{\max g, \frac{3 h-g}{2}\right\}$, so that

$$
r \geqq h_{1}^{\prime}+h_{3}^{\prime}+\max \left\{g, \frac{3 h-g}{2}\right\},
$$

which ultimately will prove 9).

We return to the problem of minimizing $r, n$ later. First we show suitable $\tilde{a}_{1}, \tilde{a}_{3}$ exist, and with $\delta \geqq \frac{h-g}{2}$.

The idea is this: by 3 ), $h_{i}^{\prime} \geqq g-h$, whence $h_{i}^{\prime}+h \geqq g$, and so $H_{i}=H \oplus H_{i}^{\prime}$ can be mapped onto $G, i=1,3$. We shall define $\tilde{a}_{1}$ so that it maps $H_{i}$ onto $G$ and takes $H_{4-i}^{\prime}$ to the copy of itself in $T=G \oplus H_{3}^{\prime} \oplus H_{1}^{\prime}$ via the identity map. Regardless of how $\tilde{a}_{1}, \tilde{a}_{3}$ map $H_{1}, H_{3}$, respectively, onto $G$, we then have $\operatorname{Im} \tilde{a}_{1} \cap \operatorname{Im} \tilde{a}_{3} \cong G, \quad \tilde{a}_{i}^{-1}(G)=H_{i}, \quad H_{1} \cap H_{3}=H, \quad H_{1}+H_{3}=K^{s}, \quad \operatorname{Im} \tilde{a}_{1}+\operatorname{Im} \tilde{a}_{3}=T$, and all the conditions we need will be satisfied provided we can define $\tilde{a}_{i}$ on $H_{i}$ so that:
a) $\tilde{a}_{i}: H_{i}$ onto $G$
b) $\operatorname{Ker} \tilde{a}_{1} \cap \operatorname{Ker} \tilde{a}_{3}=0$
c) $\delta\left(\tilde{a}_{1}, \tilde{a}_{3}\right) \geqq \frac{h-g}{2}$. 
Note that $\delta\left(a_{1}, a_{3}\right)$ will equal $\delta\left(\tilde{a}_{1}, \tilde{a}_{3}\right)$, and the kernel of $a_{i}$ will be the same as that of $\tilde{a}_{i}$ on $K^{s}$, and will be contained in $H_{i}$ and so identified with the kernel of the restricted $\tilde{a}_{i}: H_{i} \rightarrow G$.

We use general position arguments to show we can do this. Let $k_{i}=h+h_{i}^{\prime}$ $-\mathrm{g}, i=1,3$. By 3 ), $k_{i} \geqq 0$. Pick any basis $w_{1}, \ldots, w_{\mathrm{g}}$ for $G$. Pick bases $u_{11}, \ldots, u_{1 h_{1}}$ for $H_{1}$ and $u_{31}, \ldots, u_{3 h_{3}}$ for $H_{3}$ (where $h_{i}=\operatorname{dim} H_{i}=h+h_{i}^{\prime}$ ) which are in "general position" in the following precise sense: if $0 \leqq j_{i} \leqq h_{i}, i=1,3$ and $U_{i}$ is the span of a $j_{i}$-element subset of $u_{i 1}, \ldots, u_{i h_{i}}, i=1,3$, then $\operatorname{dim}_{K} U_{1} \cap U_{3}=\left(j_{1}+j_{3}\right)-s$.

To see that this is possible we must show that a $j_{1}$-dimensional subspace $U_{1}$ of $H_{1}$ in general position (i.e. off a proper closed subvariety of the appropriate Grassmannian) meets a $j_{3}$-dimensional subspace $U_{3}$ of $H_{3}$ in general position in a subspace of dimension $\left(j_{1}+j_{3}\right)-s$. This would be clear if it were not for the "tag" conditions $U_{i} \subset H_{i}$. However, because $H_{1}+H_{3}=K^{s}$ these conditions do not affect the issue. To understand this, first note that $U_{1} \cap U_{3} \subset H_{1} \cap H_{3}$ $=H$, so that $U_{1} \cap U_{3}=\left(U_{1} \cap H\right) \cap\left(U_{3} \cap H\right)$. For $U_{i}$ in general position in $H_{i}$, $U_{i} \cap H$ has general position in $H$ and dimension $j_{i}+h \dot{-}\left(h+h_{i}^{\prime}\right)=j_{i}-h_{i}^{\prime}$. Hence, $U_{1} \cap U_{3}=\left(U_{1} \cap H\right) \cap\left(U_{3} \cap H\right)$ has dimension

$$
\left[\left(j_{1}-h_{1}^{\prime}\right)+\left(j_{3}-h_{3}^{\prime}\right)\right] \div h=\left(j_{1}+j_{3}\right) \div\left(h_{1}^{\prime}+h_{3}^{\prime}+h\right)=j_{1}+j_{3} \dot{-s},
$$

as claimed.

Now define $\tilde{a}_{i}$ by mapping the first $g$ elements in $u_{i 1}, \ldots, u_{i h_{i}}$ to $w_{1}, \ldots, w_{g}$ respectively, and the remaining $h_{i}-g$ elements to 0 .

It is clear that $\tilde{a}_{1}, \tilde{a}_{3}$ map $H_{1}, H_{3}$ respectively onto $G$. The kernels are subspaces of $H_{1}, H_{3}$ respectively spanned by subsets of the $u_{i 1}, \ldots, u_{i h_{i}}$, and their dimensions are $k_{1}=h+h_{1}^{\prime}-g$ and $k_{3}=h+h_{3}^{\prime}-g$. Hence, the intersection has dimension

$$
\left(k_{1}+k_{3}\right)-s=\left(2 h-2 g+h_{1}^{\prime}+h_{3}^{\prime}\right) \div\left(h+h_{1}^{\prime}+h_{3}^{\prime}\right)=h \dot{-2} g=0
$$

since $h \leqq 2 g$.

Let $q$ be a nonnegative integer such that

i) $q \leqq \min \left\{h+h_{1}^{\prime}, h+h_{3}^{\prime}\right\}$

ii) $2 q \leqq s$.

Then, by i), $\tilde{a}_{1}\left(u_{1 i}\right)=\tilde{a}_{3}\left(u_{3 i}\right), 1 \leqq i \leqq q$, and the general position condition implies, using ii), that $\operatorname{span}\left\{u_{1 i}\right\} \cap \operatorname{span}\left\{u_{3 i}\right\}=0$, for its dimension will be $2 q \dot{-}$, showing that $u_{11}, \ldots, u_{1 q}, u_{31}, \ldots, u_{3 q}$ are independent. Hence for any such $q$ we shall have $\delta\left(\tilde{a}_{1}, \tilde{a}_{3}\right) \geqq q$. It suffices then, to complete the proof of the existence of the $\tilde{a}_{i}$, to show we can take $q \geqq \frac{h-g}{2}$. If $h=g$ there is no problem: Let $q=0$. If $h \neq g$ then since $g \leqq h+h_{i}^{\prime}, i=1,3$ we have

$$
2 g \leqq 2 h+h_{1}^{\prime}+h_{3}^{\prime}=s+h \leqq 2 s
$$

and equality can only hold if $h_{1}^{\prime}=h_{3}^{\prime}=0$ and $g=h$. Thus when $g \neq h, 2 g<2 s$ and $g<s \Rightarrow g+1 \leqq s \Rightarrow(g+1) / 2 \leqq s / 2$ and so $2[(g+1) / 2] \leqq s$. Let $q=[(g+1) / 2]$. We have $2 q \leqq s$, but also, $q \leqq g \leqq \min \left\{h+h_{1}^{\prime}, h+h_{3}^{\prime}\right\}$ by 3$)$. Thus $q=[(g+1) / 2]$ satisfies both i) and ii). But $q \geqq g / 2 \geqq(h-g) / 2$ since $h \leqq 2 g$ by 2 ). 
We already established 9) when we proved (4.2). Formula 8) shows that $n$ is minimum when $r$ is, and this value for

$$
\begin{aligned}
n & =h+h_{1}^{\prime}+h_{3}^{\prime}-\frac{g-h}{2}+2 r \\
& =h+h_{1}^{\prime}+h_{3}^{\prime}-\frac{g-h}{2}+2\left(h_{1}^{\prime}+h_{3}^{\prime}\right)+\max \{2 g, 3 h-g\} \\
& =3\left(h+h_{1}^{\prime}+h_{3}^{\prime}\right)-2 h-\frac{g-h}{2}+\max \{2 g, 3 h-g\} \\
& =3\left(h+h_{1}^{\prime}+h_{3}^{\prime}\right)+\max \left\{2 g-2 h-\frac{g-h}{2}, h-g-\frac{g-h}{2}\right\} \\
& =3\left(h+h_{1}^{\prime}+h_{3}^{\prime}\right)+\frac{3}{2} \max \{g-h, h-g\}=3\left(h+h_{1}^{\prime}+h_{3}^{\prime}\right)+\frac{3}{2}|g-h|,
\end{aligned}
$$

as claimed.

It is clear from 9) and 10) that both $r$ and $n$ are minimized by choosing $h_{1}^{\prime}$, $h_{3}^{\prime}$ as small as possible. By 3), this means $h_{1}^{\prime}=h_{3}^{\prime}=g-h$.

The formulae for $\chi(M, R / P), \chi(M, R / Q)$ are immediate from Theorem (3.7) and 6). Q.E.D.

(4.3) Theorem. Let $\mathscr{M}$ be the class of $R$-modules of finite length and finite projective dimension killed by $\mathrm{m}^{3}+\left(x_{2}, x_{4}\right) \mathfrak{m}$.

a) There exists $M \in \mathscr{M}$ such that $l(M)=15$ and $\chi(M, R / P)=-1$. Moreover, then $M^{\vee} \in \mathscr{M}$ and $\chi\left(M^{\vee}, R / P\right)=1$.

b) For $M \in \mathscr{M}$, if $l(M)<15$ then $\chi(M, R / P)=0$.

c) For every $M \in \mathscr{M}$,

$$
|\chi(M, R / P)| / l(M) \leqq 1 / 15
$$

(equivalently, $l(M) \geqq 15|\chi(M, R / P)|)$.

Proof. All the real work has already been done in the proof of Theorem (4.1). We only need to analyze the possibilities as $g$ and $h$ vary.

Let $g, h$ be nonnegative integers such that $|g-h|$ is even and let $|g-h|=2 \mu$. We consider two cases.

1) $g \geqq h$. The condition $h \leqq 2 g$ is automatic. We have $g=h+2 \mu$, and we may assume $\mu \geqq 1$. Then $g-h=2 \mu$ and we take $h_{1}^{\prime}=h_{3}^{\prime}=g-h$ so that $s=h+h_{1}^{\prime}$ $+h_{3}^{\prime}=h+2 \mu+2 \mu=h+4 \mu$. The smallest possible value for $n$ for given $\mu, h$ is then $3 s+\frac{3}{2}|g-h|=3 h+12 \mu+3 \mu=3 h+15 \mu$. Then

$$
|\chi(M, R / P)| / l(M) \leqq \mu /(3 h+15 \mu) \leqq 1 / 15
$$

(or $|\operatorname{rank} A-l(M)| / l(M) \leqq 1 / 15$ ). The best possibility is $h=0$, and $M$ of smallest length occurs for $\mu=1$.

2) $h \geqq g$. Then $h=\mathrm{g}+2 \mu$, where $\mathrm{g} \geqq 0, \mu \geqq 1$. The condition $h \leqq 2 \mathrm{~g}$ becomes $g$ $+2 \mu \leqq 2 g$ or $g \geqq 2 \mu$. Then $g-h=0$, and we should take $s=h+h_{1}^{\prime}+h_{3}^{\prime}=h=g$ 
$+2 \mu$. The smallest possible value of $n$ for fixed $\mu, g$ is $3(g+2 \mu)+\frac{3}{2}(2 \mu)=3 g$

$+9 \mu \geqq 15 \mu$, since $g \geqq 2 \mu$. Again, this shows

$$
|\chi(M, R / P)| / l(M) \leqq \mu /(3 g+9 \mu) \leqq 1 / 15 .
$$

The smallest value of $n=3 g+9 \mu$ with $\mu \neq 0$ occurs for $\mu=1, h=4, g$ $=2(g \geqq 2 \mu)$, and is, again, 15 . Q.E.D.

(4.4) Remark. The cases $g>h$ and $h>g$ are not really distinct: they correspond to $\chi(M, R / P)>0$ and $\chi(M, R / P)<0$, and so are interchanged by replacing $M$ by $M^{*}$ or $M^{\vee}$.

\section{The case $n=15$}

In this section we want to study in greater detail the most accessible of our examples: the $R$-modules $M$ of the smallest length such that $\mathrm{m}^{3}+\left(x_{2}, x_{4}\right) \mathrm{m}$ kills $M, p d_{R} M$ is finite and $\chi(M, R / P) \neq 0$. These have length 15 , and we already know by (4.3) that $\chi(M, R / P)= \pm 1$ for such an $M$. Since we are free to replace $M$ by $M^{*}$ or $M^{\vee}$, we limit ourselves to the case $\chi(M, R / P)=-1$.

Because these modules are a new breed among modules of finite projective dimension, we feel they are worthy of close scrutiny.

(5.1) Theorem. Let $M$ be an $R$-module of length 15 such that $M \in \mathscr{M}$ and $\chi(M, R / P)<0$. Then:
a) $\chi(M, R / P)=-1$
b) $\operatorname{dim}_{K} M / \mathfrak{m} M=6$
c) $\operatorname{dim}_{K} \mathrm{Ann}_{M} \mathrm{~m}=5$, and $\mathrm{Ann}_{M} \mathrm{~m}=\mathrm{m}^{2} M+\left(x_{2}, x_{4}\right) M$
d) $\operatorname{dim}_{K} \operatorname{Tor}_{1}(K, M)=17$
e) $\operatorname{dim}_{K} \operatorname{Tor}_{2}(K, M)=16$.

For any such module $M$, for a suitable identification of $M \cong K^{15}$, the matrices $A_{i}$ of the actions of the $x_{i}$ have the following form:

$$
\begin{aligned}
A_{1}= & \left.\begin{array}{lll}
5 & 4 & 6 \\
5 & {\left[\begin{array}{lll}
0 & a_{1} & c_{1} \\
0 & 0 & b_{1} \\
0 & 0 & 0
\end{array}\right]} & 5
\end{array} \quad \begin{array}{rll}
5 & 4 & 6 \\
0 & 0 & c_{2} \\
0 & 0 & 0 \\
0 & 0 & 0
\end{array}\right] \\
A_{3}=4\left[\begin{array}{llll}
5 & 4 & 6 \\
6 & a_{3} & c_{3} \\
0 & 0 & b_{3} \\
0 & 0 & 0
\end{array}\right] & A_{4}=4\left[\begin{array}{lll}
5 & 4 & 6 \\
0 & 0 & c_{4} \\
0 & 0 & 0 \\
0 & 0 & 0
\end{array}\right]
\end{aligned}
$$


(so that $a_{2}=a_{4}=0$ and $b_{2}=b_{4}=0$ ) where

$$
\begin{aligned}
& 22 \\
& a_{1}=\frac{2}{3}\left[\begin{array}{ll}
1 & 0 \\
0 & 0
\end{array}\right], \quad a_{3}=\frac{2}{3}\left[\begin{array}{ll}
0 & 1 \\
0 & 0
\end{array}\right] \\
& \begin{array}{lll}
2 & 2 & 2
\end{array} \\
& 222 \\
& b_{1}=2\left[\begin{array}{lll}
1 & 0 & 0 \\
0 & 1 & 0
\end{array}\right] \text { and } b_{3}=\begin{array}{l}
2 \\
2
\end{array}\left[\begin{array}{lll}
0 & 1 & 0 \\
0 & 0 & 1
\end{array}\right]
\end{aligned}
$$

(Matrices with the specified form automatically commute, satisfy $A_{1} A_{4}=A_{2} A_{3}$ and define a module killed by $\mathrm{m}^{3}+\left(x_{2}, x_{4}\right) \mathrm{mt}$.) The module $M$ defined by four matrices in this form satisfies $p d_{R} M<\infty$ and $\chi(M, R / P)=-1$ if and only if the entries of the four variable matrices $c_{i}$ are in sufficiently general position in the precise sense that if we write

$$
c_{i}=\frac{2}{3}\left[\begin{array}{ccc}
2 & 2 & 2 \\
c_{i 1} & c_{i 2} & c_{i 3} \\
d_{i 1} & d_{i 2} & d_{i 3}
\end{array}\right]
$$

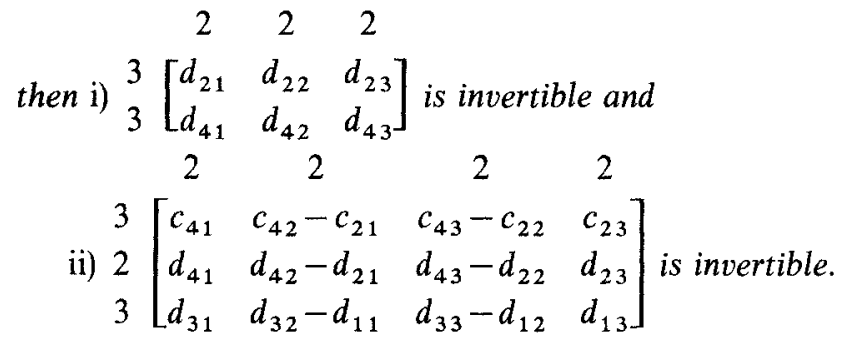

(5.2) Remark. We have parametrized the isomorphism classes of the modules $M$ in which we are interested by the points of a dense open set in $\mathbf{A}_{K}^{120}$, but this parametrization is by no means one-to-one. Two quadruples of matrices give the same isomorphism class if and only if they are simultaneously conjugate. Thus, for example, we may do any row operation involving only the third, fourth and fifth rows (to all four matrices) without changing the isomorphism class: the inverse column operation will have no effect, since the third, fourth, and fifth columns are zero. Later (Corollary 5.4) we shall impose further restrictions on the $c_{i}$.

(5.3) Remark. The numbers given in b)-e) show that a minimal resolution of such a module $M$ has the form

$$
0 \rightarrow R^{5} \rightarrow R^{16} \rightarrow R^{17} \rightarrow R^{6} \rightarrow M \rightarrow 0 .
$$

The number 17 represents the minimum number of generators for the relations on the 6 generators of $M$. By dualizing into $R$, we see that $M^{*}$ requires 5 generators and 16 relations. R. Fossum has computed the resolution in detail for one choice of $M\left[F_{2}\right]$. 
Proof of Theorem (5.2). We shall first establish a), b), c) and then the lengthy remarks following statement e). We shall then return to the calculations necessary to establish d) and e).

In the notation of (4.1) and (4.3) we have $\chi(M, R / P)=(g-h) / 2$ and so we must be in case 2) of the proof of Theorem (4.3). In order to achieve the minimum length of 15 we must have $h=4, g=2$, and $h_{1}^{\prime}=h_{3}^{\prime}=g-h=0$. Then $s$ $=h+h_{1}^{\prime}+h_{3}^{\prime}=4$,

$$
r=h_{1}^{\prime}+h_{3}^{\prime}+\max \left\{g, \frac{3}{2} h-\frac{1}{2} g\right\}=0+0+\max \{2,6-1\}=5
$$

and $t=r-\frac{g-h}{2}=5-(-1)=6$ are the values we must take to minimize $n=r+s$ $+t=15$. Thus, a module of length 15 which satisfies our requirements has only one admissible filtration $M=M_{0} \supset M_{1} \supset M_{2} \supset=0$ satisfying $\mathrm{m} M_{i} \subset M_{i+1}$, $0 \leqq i \leqq 2$ and $\left(x_{2}, x_{4}\right) M \subset M_{2},\left(x_{2}, x_{4}\right) M_{1}=0$. As observed earlier, $M_{1}=m M, M_{2}$ $=\mathrm{m}^{2} M+\left(x_{2}, x_{4}\right) M$ is always one such filtration: hence $\operatorname{dim}(M / \mathrm{m} M)=t=6$. However, when $p d M<\infty$ we may choose $M_{2}=\mathrm{Ann}_{M} \mathrm{~m}$ instead: the only problem is to see that $A_{n n} \mathfrak{m} \subset \mathfrak{m} M$. But, if not, we can choose a minimal generator $u$ of $M$ such that $\mathfrak{m} u=0$, and then $K \hookrightarrow M$ via $\overline{1} \mapsto u$ splits: the submodule of $M$ spanned by the terms other than $u$ in a minimal basis for $M$ containing $u$ will be an $R$-module complement, $M^{\prime}$, for the copy of $K$. But $M$ $=K \oplus_{R} M^{\prime}$ is impossible: $p d_{R} M<\infty$ then implies $p d_{R} K<\infty$, a contradiction.

It follows that $\mathrm{Ann}_{M} \mathrm{~m}=\mathrm{m}^{2} M+\left(x_{2}, x_{4}\right) M$, and both have dimension $r=5$.

To analyze the structure of such modules $M$ further we adopt the viewpoint and notation of the proof of Theorem (4.1). We already know that $M$ can be described by giving four matrices of the special form (3.1). From the proof of (4.1) and the discussion above we know that $\operatorname{Im} a_{1} \cap \operatorname{Im} a_{3}=G$ had dimension $g$ $=2$ and that both $a_{1}, a_{3}$ map all of $H=K^{s}=K^{4}$ into and, in fact, onto $G \cong K^{2}$. Since $\operatorname{Ker} a_{1} \cap \operatorname{Ker} a_{3}=0$ we see that $\operatorname{Ker} a_{1} \cong \operatorname{Ker} a_{3} \cong K^{2}$ and $K^{4}$ $=\operatorname{Ker} a_{1} \oplus \operatorname{Ker} a_{3}$. Choose bases for $K^{4}, K^{5}$ respectively so that $a_{1}$ maps the first two standard basis vectors to the first two standard basis vectors for $K^{5}$ (set up the basis for $K^{5}$ so that $G=K e_{1}+K e_{2}$ ) and $a_{1}$ kills the last two, while $a_{3}$ kills the first two standard basis vectors for $K^{4}$ and maps the last two to the standard basis for $G \cong K^{2} \subset K^{5}$. These choices will guarantee

$$
a_{1}=\frac{2}{3}\left[\begin{array}{ll}
2 & 2 \\
3 & 0 \\
0 & 0
\end{array}\right] \text { and } a_{3}=\begin{aligned}
2 \\
3
\end{aligned}\left[\begin{array}{ll}
0 & 1 \\
0 & 0
\end{array}\right]
$$

where the numbers alongside the matrices are used to indicate the sizes of the blocks. Let $b_{1}=2\left[\begin{array}{l}b_{1}^{\prime} \\ b_{1}^{\prime \prime}\end{array}\right]$ and $b_{3}=\frac{2}{2}\left[\begin{array}{l}b_{3}^{\prime} \\ b_{3}^{\prime \prime}\end{array}\right]$. The condition $a_{1} b_{3}=a_{3} b_{1}$ becomes $b_{1}^{\prime \prime}=b_{3}^{\prime}$ : call their common value $b^{*}$. After deleting the rows and columns consisting entirely of zeros, the matrices $\left[\begin{array}{ll}A_{1} & A_{2} \\ A_{3} & A_{4}\end{array}\right]$ and $\left[\begin{array}{rr}A_{4} & -A_{2} \\ -A_{3} & A_{1}\end{array}\right]$ become 


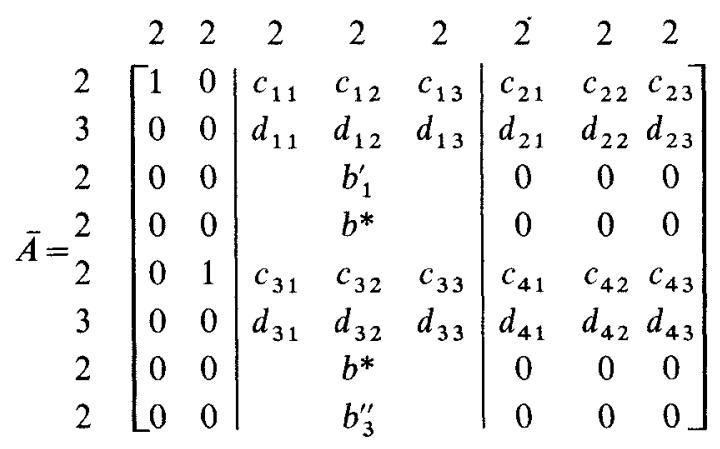

and

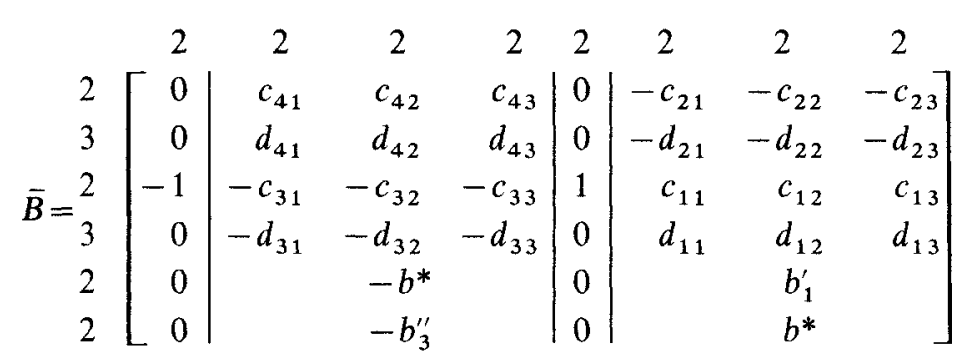

respectively. The ranks of the these two can be seen to be at most 16 and 14 , respectively, and so $M$ will have finite projective dimension precisely when these ranks are 16 and 14, respectively. We delete the rows and columns of the two 1's which occur in $\bar{A}$ : the rank of $A$ is $2+2+$ the rank of the resulting matrix, which is

$$
6\left[\begin{array}{ccc|ccc}
d_{11} & d_{12} & d_{13} & d_{21} & d_{22} & d_{23} \\
d_{31} & d_{32} & d_{33} & d_{41} & d_{42} & d_{43} \\
\hline b_{1}^{\prime} & & & \\
b^{*} & & & 0 \\
b_{3}^{\prime \prime} & & &
\end{array}\right]
$$

and this matrix will have the required rank, 12 , if and only if both

$$
\left[\begin{array}{l}
b_{1}^{\prime} \\
b^{*} \\
b_{3}^{\prime \prime}
\end{array}\right] \text { and }\left[\begin{array}{lll}
d_{21} & d_{22} & d_{23} \\
d_{41} & d_{42} & d_{43}
\end{array}\right]
$$

are invertible. But then we may choose a basis for $K^{6}$ so that the first of these becomes the identity, and this gives the forms stated for the $A_{i}$ in the theorem as well as condition i). It remains to verify that the second matrix $\bar{B}$ has rank 14 if and only if, in addition, condition ii) holds. Deleting the first column (actually, two columns) of $\bar{B}$, which are redundant, and substituting for $b_{1}^{\prime}, b^{*}$, $b_{3}^{\prime \prime}$, we obtain 


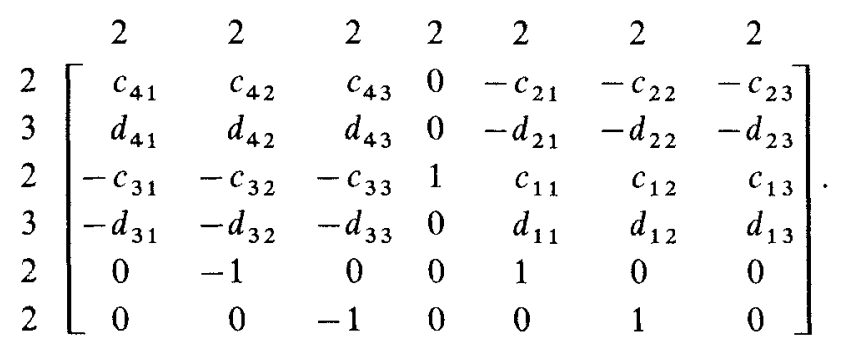

If we add the second column to the fifth and the third column to the sixth, and then delete the rows and columns of the three l's (each of which is either the only nonzero element in its row or in its column), we see that $B$ has rank $2+2$ $+2+$ the rank of

$$
\begin{aligned}
& 2 \\
& 3
\end{aligned}\left[\begin{array}{rrrr}
c_{41} & c_{42}-c_{21} & 2 & 2 \\
d_{41} & d_{42}-d_{21} & d_{43}-d_{22} & -c_{23} \\
-d_{31} & -d_{32}+d_{11} & -d_{33}+d_{12} & d_{13}
\end{array}\right],
$$

which must have rank 8 , i.e. be invertible, in order that $\bar{B}$ have rank 14 . But switching the signs on both the last row and the last column yields the matrix given in ii).

It remains to establish d) and e). We first note that the resolution of $K$ over $R$ has the form:

$$
\ldots \longrightarrow R^{7} \stackrel{\psi}{\longrightarrow} R^{4} \stackrel{\phi}{\longrightarrow} R \longrightarrow K \longrightarrow 0
$$

where the matrices of the maps $\phi, \psi$ are then transposes of the matrices

and

$$
\left[\begin{array}{llll}
x_{1} & x_{2} & x_{3} & x_{4}
\end{array}\right]
$$

$$
L_{0}=\left[\begin{array}{cccc}
x_{2} & -x_{1} & 0 & 0 \\
x_{3} & 0 & -x_{1} & 0 \\
x_{4} & 0 & 0 & -x_{1} \\
0 & x_{3} & -x_{2} & 0 \\
0 & x_{4} & 0 & -x_{2} \\
0 & 0 & x_{4} & -x_{3} \\
0 & 0 & x_{2} & -x_{1}
\end{array}\right]
$$

respectively. (The first six rows are Koszul relations.)

If we apply $\otimes_{K} M$ we see that $\operatorname{Tor}_{1}^{R}(K, M)$ is the homology, at the center spot, of

whence

$$
M^{\urcorner} \stackrel{\psi \otimes \mathrm{id}_{M}}{\longrightarrow} M^{4} \stackrel{\phi \otimes \mathrm{id}_{M}}{\longrightarrow} M
$$

$$
\begin{aligned}
\operatorname{dim}_{K} \operatorname{Tor}_{1}^{R}(K, M) & =\operatorname{dim} M^{4}-\operatorname{rank} \psi \otimes \mathrm{id}_{M}-\operatorname{rank} \phi \otimes \mathrm{id}_{M} \\
& =60-\operatorname{rank} \psi \otimes \mathrm{id}_{M}-9=51-\operatorname{rank} \psi \otimes \mathrm{id}_{M}
\end{aligned}
$$

since

$$
\begin{aligned}
\operatorname{rank} \phi \otimes \operatorname{id}_{M} & =\operatorname{dim}_{K}\left(\operatorname{Im}\left(\phi \otimes \operatorname{id}_{M}\right)\right)=\operatorname{dim}_{K} \mathrm{mM} \\
& =l(M)-l(M / \mathrm{m} M)=15-6=9 .
\end{aligned}
$$


To complete the argument we need to show that rank $\psi \otimes \mathrm{id}_{M}$ is 34 . If we identify $M$ with $K^{15}$ we may replace $\psi \otimes \mathrm{id}_{M}$ by a $60 \times 105$ matrix $L^{t}$ which arises from $L_{0}^{t}$ by replacing each $x_{i}$ by the matrix $A_{i}$ of the action of $x_{i}$ on $M$ $=K^{15}$. In fact, we may use $\overline{A_{i}}$ instead of $A_{i}$, omitting the first five columns and last six rows of each $A_{i}$, all of which are 0 . The result is shown below, in block form. All blocks are $2 \times 2$ except for the $d$ 's, which are $3 \times 2$, and the $0^{\prime}$ entries, which are $3 \times 2$ blocks of 0 's. The numbers shown are simply to keep track of rows and columns, not block sizes. The resulting matrix is 63 $\times 40$, but is presented as a $28 \times 20$ matrix of $2 \times 2$ and $3 \times 2$ blocks.

Each of the entries in the spots $(1,6),(5,2),(13,7)$, and $(21,17)$ is \pm 1 and is the only nonzero entry in its column. Each of the entries in the spots $(3,8)$, $(4,9),(11,18),(12,19),(16,10)$, and $(24,20)$ is \pm 1 and is the only nonzero entry in its row. These 10 (boldface) entries are in 10 distinct rows and 10 distinct columns. If we delete the rows and columns of these entries the rank will drop by $10 \cdot 2=20$. If we also delete all the rows and columns which are identically 0 after these deletions, namely rows $15,17,18,19,20,27$ and 28

\begin{tabular}{|c|c|c|c|c|c|c|c|c|c|c|c|c|c|c|c|c|c|c|c|c|}
\hline & 1 & 2 & 3 & 4 & 5 & 6 & 7 & 8 & 9 & 10 & 11 & 12 & 13 & 14 & 15 & 16 & 17 & 18 & 19 & 20 \\
\hline 1 & $\tau_{0}$ & 0 & $c_{21}$ & $c_{22}$ & $c_{23}$ & -1 & 0 & $-c_{11}$ & $-c_{12}$ & $-c_{13}$ & 0 & 0 & 0 & 0 & 0 & 0 & 0 & 0 & 0 & 0 \\
\hline 2 & $0^{\prime}$ & $0^{\prime}$ & $d_{21}$ & $d_{22}$ & $d_{23}$ & $\sigma^{\prime}$ & $0^{\prime}$ & $-d_{11}$ & $-d_{12}$ & $-d_{13}$ & $0^{\prime}$ & $0^{\prime}$ & $0^{\prime}$ & $0^{\prime}$ & $0^{\prime}$ & $0^{\prime}$ & $0^{\prime}$ & $0^{\prime}$ & $0^{\prime}$ & $0^{\prime}$ \\
\hline 3 & 0 & 0 & 0 & 0 & 0 & 0 & 0 & -1 & 0 & 0 & 0 & 0 & 0 & 0 & 0 & 0 & 0 & 0 & 0 & 0 \\
\hline 4 & 0 & 0 & 0 & 0 & 0 & 0 & 0 & 0 & -1 & 0 & 0 & 0 & 0 & 0 & 0 & 0 & 0 & 0 & 0 & 0 \\
\hline 5 & 0 & 1 & $c_{31}$ & $c_{32}$ & $c_{33}$ & 0 & 0 & 0 & 0 & 0 & -1 & 0 & $-c_{11}$ & $-c_{12}$ & $-c_{13}$ & 0 & 0 & 0 & 0 & 0 \\
\hline 6 & $0^{\prime}$ & $0^{\prime}$ & $d_{31}$ & $d_{32}$ & $d_{33}$ & $\sigma$ & $0^{\prime}$ & $\sigma^{\prime}$ & $\sigma^{\prime}$ & $0^{\prime}$ & $0^{\prime}$ & $0^{\prime}$ & $-d_{11}$ & $-d_{12}$ & $-d_{13}$ & $0^{\prime}$ & $0^{\prime}$ & $0^{\prime}$ & $0^{\prime}$ & $0^{\prime}$ \\
\hline 7 & 0 & 0 & 0 & 1 & 0 & 0 & 0 & 0 & 0 & 0 & 0 & 0 & -1 & 0 & 0 & 0 & 0 & 0 & 0 & 0 \\
\hline 8 & 0 & 0 & 0 & 0 & 1 & 0 & 0 & 0 & 0 & 0 & 0 & 0 & 0 & -1 & 0 & 0 & 0 & 0 & 0 & 0 \\
\hline 9 & 0 & 0 & $c_{41}$ & $c_{42}$ & $c_{43}$ & 0 & 0 & 0 & 0 & 0 & 0 & 0 & 0 & 0 & 0 & -1 & 0 & $-c_{11}$ & $-c_{12}$ & $-c_{13}$ \\
\hline 10 & $0^{\prime}$ & $\sigma^{\prime}$ & $d_{41}$ & $d_{42}$ & $d_{43}$ & $\gamma$ & $0^{\prime}$ & $0^{\prime}$ & $0^{\prime}$ & $0^{\prime}$ & $0^{\prime}$ & $0^{\prime}$ & $0^{\prime}$ & $0^{\prime}$ & $0^{\prime}$ & $0^{\prime}$ & $0^{\prime}$ & $0^{\prime}$ & $-d_{12}$ & $-d_{13}$ \\
\hline 11 & 0 & 0 & 0 & 0 & 0 & 0 & 0 & 0 & 0 & 0 & 0 & 0 & 0 & 0 & 0 & 0 & 0 & -1 & 0 & 0 \\
\hline 12 & 0 & 0 & 0 & 0 & 0 & 0 & 0 & 0 & 0 & 0 & 0 & 0 & 0 & 0 & 0 & 0 & 0 & 0 & -1 & 0 \\
\hline 13 & 0 & 0 & 0 & 0 & 0 & 0 & 1 & $c_{31}$ & $c_{32}$ & $c_{33}$ & 0 & 0 & $-c_{21}$ & $-c_{22}$ & $-c_{23}$ & 0 & 0 & 0 & 0 & 0 \\
\hline 14 & $0^{\prime}$ & $0^{\prime}$ & $0^{\prime}$ & $0^{\prime}$ & $0^{\prime}$ & $\sigma^{\prime}$ & $0^{\prime}$ & $d_{31}$ & $d_{32}$ & $d_{33}$ & $0^{\prime}$ & $0^{\prime}$ & $-d_{21}$ & $-d_{22}$ & $-d_{23}$ & $0^{\prime}$ & $0^{\prime}$ & $0^{\prime}$ & $0^{\prime}$ & $0^{\prime}$ \\
\hline 15 & 0 & 0 & 0 & 0 & 0 & 0 & 0 & 0 & 1 & 0 & 0 & 0 & 0 & 0 & 0 & 0 & 0 & 0 & 0 & 0 \\
\hline 16 & 0 & 0 & 0 & 0 & 0 & 0 & 0 & 0 & 0 & 1 & 0 & 0 & 0 & 0 & 0 & 0 & 0 & 0 & 0 & 0 \\
\hline 17 & 0 & 0 & 0 & 0 & 0 & 0 & 0 & $c_{41}$ & $c_{42}$ & $c_{43}$ & 0 & 0 & 0 & 0 & 0 & 0 & 0 & $-c_{21}$ & $-c_{22}$ & $-c_{23}$ \\
\hline 18 & $0^{\prime}$ & $0^{\prime}$ & $0^{\prime}$ & $0^{\prime}$ & $0^{\prime}$ & $\sigma^{\prime}$ & $0^{\prime}$ & $d_{41}$ & $d_{42}$ & $d_{43}$ & $\sigma^{\prime}$ & $0^{\prime}$ & $\sigma^{\prime}$ & $0^{\prime}$ & $0^{\prime}$ & $0^{\prime}$ & $0^{\prime}$ & $-d_{21}$ & $-d_{22}$ & $-d_{23}$ \\
\hline 19 & 0 & 0 & 0 & 0 & 0 & 0 & 0 & 0 & 0 & 0 & 0 & 0 & 0 & 0 & 0 & 0 & 0 & 0 & 0 & 0 \\
\hline 20 & 0 & 0 & 0 & 0 & 0 & 0 & 0 & 0 & 0 & 0 & 0 & 0 & 0 & 0 & 0 & 0 & 0 & 0 & 0 & 0 \\
\hline 21 & 0 & 0 & 0 & 0 & 0 & 0 & 0 & 0 & 0 & 0 & 0 & 0 & $c_{41}$ & $c_{42}$ & $c_{43}$ & 0 & -1 & $-c_{31}$ & $-c_{32}$ & $-c_{33}$ \\
\hline 22 & $0^{\prime}$ & $0^{\prime}$ & $0^{\prime}$ & $0^{\prime}$ & $\sigma^{\prime}$ & $\sigma^{\prime}$ & $0^{\prime}$ & $0^{\prime}$ & $\sigma^{\prime}$ & $0^{\prime}$ & $\sigma^{\prime}$ & $0^{\prime}$ & $d_{41}$ & $d_{42}$ & $d_{43}$ & $0^{\prime}$ & $\sigma^{\prime}$ & $-d_{31}$ & $-d_{32}$ & $-d_{33}$ \\
\hline 23 & 0 & 0 & 0 & 0 & 0 & 0 & 0 & 0 & 0 & 0 & 0 & 0 & 0 & 0 & 0 & 0 & 0 & 0 & -1 & 0 \\
\hline 24 & 0 & 0 & 0 & 0 & 0 & 0 & 0 & 0 & 0 & 0 & 0 & 0 & 0 & 0 & 0 & 0 & 0 & 0 & 0 & -1 \\
\hline 25 & 0 & 0 & 0 & 0 & 0 & 0 & 0 & 0 & 0 & 0 & 0 & 0 & $c_{21}$ & $c_{22}$ & $c_{23}$ & -1 & 0 & $-c_{11}$ & $-c_{12}$ & $-c_{13}$ \\
\hline 26 & $0^{\prime}$ & $0^{\prime}$ & $0^{\prime}$ & $0^{\prime}$ & $\sigma^{\prime}$ & $\sigma^{\prime}$ & $0^{\prime}$ & $0^{\prime}$ & $0^{\prime}$ & $\sigma^{\prime}$ & $0^{\prime}$ & $\sigma^{\prime}$ & $d_{21}$ & $d_{22}$ & $d_{23}$ & 0 & 0 & $-d_{11}$ & $-d_{12}$ & $-d_{13}$ \\
\hline 27 & 0 & 0 & 0 & 0 & 0 & 0 & 0 & 0 & 0 & 0 & 0 & 0 & 0 & 0 & 0 & 0 & 0 & -1 & 0 & 0 \\
\hline 28 & 0 & 0 & 0 & 0 & 0 & 0 & 0 & 0 & 0 & 0 & 0 & 0 & 0 & 0 & 0 & 0 & 0 & 0 & -1 & 0 \\
\hline
\end{tabular}


and columns 1,11 , and 12 , we obtain

\begin{tabular}{|c|c|c|c|c|c|c|c|}
\hline & 1 & 2 & 3 & 4 & 5 & 6 & 7 \\
\hline 1 & {$\left[d_{21}\right.$} & $d_{22}$ & $d_{23}$ & $0^{\prime}$ & $0^{\prime}$ & $0^{\prime}$ & $0^{\prime}$ \\
\hline 2 & $d_{31}$ & $d_{32}$ & $d_{33}$ & $-d_{11}$ & $-d_{12}$ & $-d_{13}$ & $0^{\prime}$ \\
\hline 3 & 0 & 1 & 0 & -1 & 0 & 0 & 0 \\
\hline 4 & 0 & 0 & 1 & 0 & -1 & 0 & 0 \\
\hline 5 & $c_{41}$ & $c_{42}$ & $c_{43}$ & 0 & 0 & 0 & -1 \\
\hline 6 & $d_{41}$ & $d_{42}$ & $d_{43}$ & $0^{\prime}$ & $0^{\prime}$ & $0^{\prime}$ & $0^{\prime}$ \\
\hline 7 & $0^{\prime}$ & $0^{\prime}$ & $0^{\prime}$ & $-d_{21}$ & $-d_{22}$ & $-d_{23}$ & $0^{\prime}$ \\
\hline 8 & $0^{\prime}$ & $0^{\prime}$ & $0^{\prime}$ & $d_{41}$ & $d_{42}$ & $d_{43}$ & $0^{\prime}$ \\
\hline 0 & 0 & 0 & 0 & $c_{21}$ & $c_{22}$ & $c_{23}$ & -1 \\
\hline & $0^{\prime}$ & $0^{\prime}$ & $0^{\prime}$ & $d_{21}$ & $d_{22}$ & $d_{23}$ & $0^{\prime}$ \\
\hline
\end{tabular}

and it will suffice to show that the matrix has rank 14 . We may delete row 7 which is the negative of row 10, and we may also add the second colum to the fourth and the third to the fifth. This yields:

\begin{tabular}{|c|c|c|c|c|c|c|c|}
\hline & 1 & 2 & 3 & 4 & 5 & 6 & 7 \\
\hline 1 & {$\left[d_{21}\right.$} & $d_{22}$ & $d_{23}$ & $d_{22}$ & $d_{23}$ & $0^{\prime}$ & $0^{7}$ \\
\hline 2 & $d_{31}$ & $d_{32}$ & $d_{33}$ & $d_{32}-d_{11}$ & $d_{33}-d_{12}$ & $-d_{13}$ & $0^{\prime}$ \\
\hline 3 & 0 & 1 & 0 & 0 & 0 & 0 & 0 \\
\hline 4 & 0 & 0 & 1 & 0 & 0 & 0 & .0 \\
\hline 5 & $c_{41}$ & $c_{42}$ & $c_{43}$ & $c_{42}$ & $c_{43}$ & 0 & -1 \\
\hline 6 & $d_{41}$ & $d_{42}$ & $d_{43}$ & $d_{42}$ & $d_{43}$ & $0^{\prime}$ & $0^{\prime}$ \\
\hline 7 & $0^{\prime}$ & $0^{\prime}$ & $0^{\prime}$ & $d_{41}$ & $d_{42}$ & $d_{43}$ & $0^{\prime}$ \\
\hline 8 & $0^{\prime}$ & $0^{\prime}$ & $0^{\prime}$ & $c_{21}$ & $c_{22}$ & $c_{23}$ & -1 \\
\hline 9 & $0^{\prime}$ & $0^{\prime}$ & $0^{\prime}$ & $d_{21}$ & $d_{22}$ & $d_{23}$ & $0^{\prime}$ \\
\hline
\end{tabular}

Deleting the second and third rows and columns will decrease the rank by 4 . If we then subtract the next to last row (numbered 8 above) from the row now numbered 5 above, we may then delete the next to last row and the last column, which will drop the rank by 2 . We will then need to show that the result,

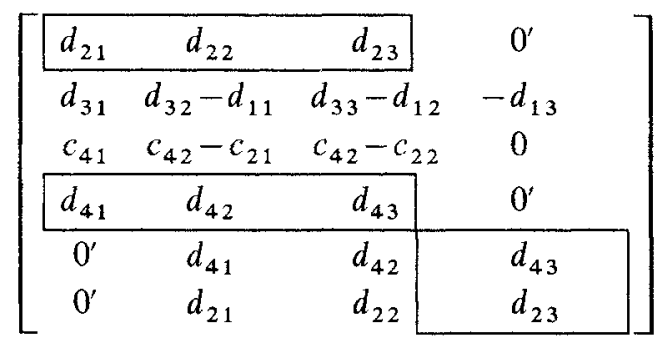


which is $17 \times 8$, has rank $14-4-2=8$. This follows because i), the submatrix

$$
\left[\begin{array}{lll}
d_{21} & d_{22} & d_{23} \\
d_{41} & d_{42} & d_{43}
\end{array}\right]
$$

is invertible, rank 6 , while the submatrix

$$
\left[\begin{array}{l}
d_{43} \\
d_{23}
\end{array}\right]
$$

has rank 2: it is, after row permutation, the last two columns of the invertible 6 $\times 6$ matrix above, which are obviously independent. This completes the proof of d).

Finally, e) follows from the fact that the alternating sum of the Betti numbers must be 0 . Q.E.D.

Note that by replacing $M$ by $M^{\vee}$ we obtain a module with essentially the same properties as $M$ (length 15 , finite projective dimension, killed by $\mathrm{m}^{3}$ $\left.+\left(x_{2}, x_{4}\right) \mathrm{m}\right)$ but such that $\chi\left(M^{\vee}, R / P\right)=1$. The Betti numbers for $M^{\vee}$ are those of $M$, but in reverse order.

(5.4) Corollary. With notation as in Theorem (5.1), for every module $M$ of length 15 killed by $\mathrm{m}^{3}+\left(x_{2}, x_{4}\right) \mathrm{m}$ such that $\chi(M, R / P)=-1$, a basis may be chosen for $M$ so that the matrices $c_{i}$ described in (5.1) satisfy the following additional conditions:

a) $c_{1 i}=c_{3 i}=0, \quad 1 \leqq i \leqq 3$

b) $d_{11}=d_{12}=0$

c) $\left[d_{21} d_{22} d_{23}\right]$ is in reduced row echelon form. (This $3 \times 6$ matrix will have rank 3.)

We then have:

$$
A_{1}=\begin{gathered}
5 \\
3 \\
2 \\
2
\end{gathered}\left[\begin{array}{c|ccccc}
0 & 1 & 0 & 0 & 0 & 0 \\
0 & 0 & 0 & 0 & 0 & d_{13} \\
\hline 0 & 0 & 0 & 1 & 0 & 0 \\
0 & 0 & 0 & 0 & 1 & 0 \\
\hline 0 & 0 & 0 & 0 & 0 & 0
\end{array}\right]
$$$$
A_{2}=\begin{gathered}
5 \\
2 \\
3 \\
2 \\
6 \\
6
\end{gathered}\left[\begin{array}{c|ccccc}
0 & 0 & 0 & c_{21} & c_{22} & c_{23} \\
0 & 0 & 0 & d_{21} & d_{22} & d_{23} \\
\hline 0 & 0 & 0 & 0 & 0 & 0 \\
0 & 0 & 0 & 0 & 0 & 0 \\
\hline 0 & 0 & 0 & 0 & 0 & 0
\end{array}\right]
$$

$$
A_{3}=\begin{gathered}
5 \\
2 \\
3 \\
2 \\
2 \\
6
\end{gathered}\left[\begin{array}{c|ccccc}
0 & 0 & 1 & 0 & 0 & 0 \\
0 & 0 & 0 & d_{31} & d_{32} & d_{33} \\
\hline 0 & 0 & 0 & 0 & 1 & 0 \\
0 & 0 & 0 & 0 & 0 & 1 \\
\hline 0 & 0 & 0 & 0 & 0 & 0
\end{array}\right]
$$

$$
A_{4}=\begin{gathered}
5 \\
2 \\
3 \\
2 \\
6
\end{gathered}\left[\begin{array}{c|cc|ccc}
0 & 0 & 0 & c_{41} & c_{42} & c_{43} \\
0 & 0 & 0 & d_{41} & d_{42} & d_{43} \\
\hline 0 & 0 & 0 & 0 & 0 & 0 \\
0 & 0 & 0 & 0 & 0 & 0 \\
\hline 0 & 0 & 0 & 0 & 0 & 0
\end{array}\right]
$$


and conditions i), ii) of Theorem (5.1) become:

i) $\left[\begin{array}{lll}d_{21} & d_{22} & d_{23} \\ d_{41} & d_{42} & d_{43}\end{array}\right]=A_{1}$ is invertible and

ii) $\left[\begin{array}{cccc}c_{41} & c_{42}-c_{21} & c_{43}-c_{22} & c_{23} \\ d_{41} & d_{42}-d_{21} & d_{43}-d_{22} & d_{23} \\ d_{31} & d_{32} & d_{33} & d_{13}\end{array}\right]=A_{2}$ is invertible.

(Whenever i), ii) are satisfied, $p d M<\infty, l(M)=15$ and $\chi(M, R / P)=-1$.)

Proof. First, we can arrange that $c_{11}=c_{12}=c_{13}=0$ by subtracting multiples of the sixth and seventh columns from the last six columns: the inverse matrix acting on rows adds multiples of the last six rows, which are 0 , to the sixth and seventh rows, with no effect. (Note that each operation is performed on all four matrices simultaneously.) We may use the eighth and ninth columns in a similar way to guarantee that $c_{31}=c_{32}=c_{33}=0$.

We may then use row operations involving the sixth and seventh rows to guarantee that $d_{11}=0$ (the inverse column operations are "harmless"), and, similarly, we may use row operations involving the eighth and ninth rows to guarantee that $d_{12}=0$ ( $d_{33}$ changes, but that doesn't matter).

The matrix $\left[d_{21} d_{22} d_{23}\right]$ must have rank 3 , because the matrix listed in i) is invertible. Since row operations within the third, fourth and fifth rows preserve all the zeros we have introduced, while the corresponding columns are 0 , we may assume $\left[d_{21} d_{22} d_{31}\right]$ is in reduced row echelon form. The conditions i) and ii) listed are the same as in Theorem (5.1), simply taking account of the zeros we have introduced. Q.E.D.

The parametrization of the isomorphism classes is still not one-to-one, even with these restrictions.

(5.5) Corollary. For every field $K$, finite or infinite, there exists an $R$-module $M$ of length 15 killed by $\mathrm{m}^{3}+\left(x_{2}, x_{4}\right) m$ of finite projective dimension such that $\chi(M, R / P)=-1$. In fact we may take $c_{41}=1, c_{21}=c_{22}=c_{23}=c_{42}=c_{43}=0$, and

$$
d_{13}=d_{43}=\left[\begin{array}{ll}
0 & 0 \\
1 & 0 \\
0 & 1
\end{array}\right], \quad d_{21}=\left[\begin{array}{ll}
1 & 0 \\
0 & 1 \\
0 & 0
\end{array}\right], \quad d_{22}=\left[\begin{array}{ll}
0 & 0 \\
0 & 0 \\
1 & 0
\end{array}\right],
$$
$d_{23}=d_{31}=d_{32}=d_{41}=0$, and $d_{33}=d_{42}=\left[\begin{array}{ll}0 & 1 \\ 0 & 0 \\ 0 & 0\end{array}\right]$, and all other choices as in
(5.4).

Proof. Since we are constructing $M$ explicitly, the result does not depend on the general position arguments used earlier and we may allow $K$ to be finite. With these choices $\Delta_{1}$ is a $6 \times 6$ identity matrix, while $\Delta_{2}$ is the direct sum of a $2 \times 2$ identity, the upper triangular $4 \times 4$ matrix 


$$
\left[\begin{array}{rrrr}
-1 & 1 & 0 & 0 \\
0 & -1 & 1 & 0 \\
0 & 0 & -1 & 1 \\
0 & 0 & 0 & 1
\end{array}\right]
$$

and another $2 \times 2$ identity matrix. Hence, $\Delta_{1}$ and $\Delta_{2}$ are both invertible. Q.E.D.

\section{Some positive results}

(6.1) Theorem. Let $M$ be an R-module of finite length and suppose that at least one of the following conditions holds:
a) $M \cong M^{*}\left(=\operatorname{Hom}_{K}(M, K)\right)$
b) $\mathrm{m}^{2} M=0$
c) $x_{i} M=0$ for some $i, 1 \leqq i \leqq 4$
d) $l(M) \leqq 7$.

Then $l\left(M \otimes_{R} P\right) \geqq l(M)$ and $l\left(M \otimes_{R} Q\right) \geqq l(M)$.

Moreover, if $p d_{R} M<\infty$ and any of the above conditions holds or

$\left.c^{\prime}\right) M$ is killed by an element $x \in \mathrm{m}-\mathrm{m}^{2}$ then $\chi(M, R / P)=\chi(M, R / Q)=0$.

Proof. From the exact sequence

$$
0 \rightarrow Q \rightarrow R^{2} \rightarrow P \rightarrow 0
$$

we have that $2 \chi(M, R)=\chi(M, P)+\chi(M, Q)$ or $2 l(M)=l(M \otimes P)+l(M \otimes Q)$ when $p d_{R} M<\infty$. Thus $(l(M \otimes P)-l(M))+(l(M \otimes Q)-l(M))=0$ and if both terms in parentheses are nonnegative then both must vanish. Moreover, $\chi(M, R / P)$ $=\chi(M, R)-\chi(M, P)=l(M)-l(M \otimes P)$ and similarly for $Q$. Thus, the first part of the theorem implies the second part, except for the statement referring to hypothesis $\mathrm{c}^{\prime}$ ). But if $\mathrm{c}^{\prime}$ ) holds and we have $x \in \operatorname{Ann} M, x \in \mathrm{m}-\mathrm{m}^{2}$, and $M$ is of finite projective dimension over $R / x R$, then since $\bar{R}=R / x R$ has dimension 2 the Grothendieck group of modules of finite length and finite projective dimension is generated by the classes of the modules $\bar{R} /(\bar{y}, \bar{z})$ where $\bar{y}, \bar{z}$ is an $\bar{R}$ sequence. But then the class of $M$ in the corresponding Grothendieck group for $R$ lies in the subgroup generated by the classes $R /(x, y, z)$, when $x, y, z$ is an $R$-sequence, and the multiplicities conjecture is known for modules with this form. See [L].

It remains to show that if any of a)-d) holds then $l(M \otimes Q) \geqq l(M)$ : the proof for $P$ is entirely similar.

We use the notation of Proposition (2.8): $A_{i}$ is the matrix of the action of $x_{i}$. By (2.8), what we must show is that if the $A_{i}$ commute and $A_{1} A_{4}=A_{2} A_{3}$, then a) or b) or c) or d) implies rank $A \leqq n$, where

$$
A=\left[\begin{array}{ll}
A_{1} & A_{2} \\
A_{3} & A_{4}
\end{array}\right] \text {. }
$$


We first do the case where c) holds. Assume that $x_{i} M=0$ : this means that $A_{i}=0$. Let $A_{j}$ be the matrix in the opposite corner of $A$, i.e. $\{i, j\}=\{2,3\}$ or $\{1,4\}$. There is no loss of generality in assuming $K$ is infinite. Consider the matrix $A_{\lambda}$ obtained by replacing $A_{j}$ by $A_{j}+\lambda I, \lambda \in K$. It will suffice to show that when $A_{j}+\lambda I$ is invertible, then $\operatorname{rank} A_{\lambda} \leqq n$ : for then, the $n+1$ size minors of $A_{\lambda}$, viewed as polynomials in $\lambda$, vanish for all but finitely many values of $\lambda$, and this means they vanish for all values of $\lambda$, including $\lambda=0$. But then, to complete the proof, it suffices to observe:

(6.2) Lemma. If $A_{1}, A_{2}, A_{3}, A_{4}$ are commuting $n \times n$ matrices such that $A_{1} A_{4}$ $=A_{2} A_{3}$ and one of them is invertible, then rank $A=n$, where

$$
A=\left[\begin{array}{ll}
A_{1} & A_{2} \\
A_{3} & A_{4}
\end{array}\right] .
$$

Proof. We do the case where $A_{1}$ is invertible for definiteness: the proofs in the other cases are essentially the same. The idea is to subtract $A_{2} A_{1}^{-1}$ times the "first" ( $n$-fold) column from the second ( $n$-fold) column. This produces

$$
\left[\begin{array}{cc}
A_{1} & 0 \\
A_{3} & A_{4}-A_{2} A_{1}^{-1} A_{3}
\end{array}\right]
$$

But since $A_{1} A_{4}=A_{2} A_{3}$ and the matrices commute we have $A_{4}-A_{2} A_{1}^{-1} A_{3}=0$, and since $A_{1}$ is invertible the rank of

$$
\left[\begin{array}{ll}
A_{1} & 0 \\
A_{3} & 0
\end{array}\right]
$$

is $n$. Q.E.D.

We now return to the rest of the proof of Theorem (6.1). Assume a), that $M \cong M^{*}$. Then from (2.8) we have $l(M \otimes P)=l(M \otimes Q)$. From the exact sequence

$$
0 \rightarrow Q \rightarrow R^{2} \rightarrow P \rightarrow 0
$$

we obtain:

$$
M \otimes Q \stackrel{\phi}{\longrightarrow} M^{2} \rightarrow M \otimes P \rightarrow 0
$$

whence, although $\phi$ may have a kernel, we still obtain

$$
l(M \otimes Q)+l(M \otimes P) \geqq 2 l(M) .
$$

Since $l(M \otimes Q)=l(M \otimes P)$, we now have the desired result.

Next, assume b), $\mathrm{m}^{2} M=0$. Choose a $K$-vector space basis $v_{1}, \ldots, v_{n}$ for $M$ such that $v_{1}, \ldots, v_{h}$ is a basis for $\mathrm{m} M$. Let $k=n-h$. Then all of the matrices $A_{i}$ have block form

$$
A_{i}=\begin{array}{cc}
h & k \\
k & {\left[\begin{array}{cc}
0 & d_{i} \\
0 & 0
\end{array}\right]}
\end{array}
$$


where $d_{i}$ is an $h \times k$ block, and so

$$
\operatorname{rank}\left[\begin{array}{ll}
A_{1} & A_{2} \\
A_{3} & A_{4}
\end{array}\right]=\operatorname{rank}\left[\begin{array}{ll}
d_{1} & d_{2} \\
d_{3} & d_{4}
\end{array}\right] \leqq \min \{2 h, 2 k\} \leqq n
$$

since $h+k=n$.

It remains to handle the case where $n=l(M) \leqq 7$. Because the length of the argument, which includes a tedious case analysis, is, in our judgment, disproportionate to the interest of the result, we have given it in an Appendix [DHM] to this paper. This Appendix is available as an unpublished "preprint" and may be obtained by writing to one of the authors. Thus, for the remainder of the argument we simply refer to [DHM].

We note that the first author has shown in $\left[\mathrm{D}_{1}\right]$ that if $R$ is local Gorenstein ring of dimension $3, M$ has finite length and finite projective dimension, and $M \cong \operatorname{Ext}^{3}(M, R)$, then $\chi(M, N)=0$ for all finitely generated modules $N$ with $\operatorname{dim} N \leqq 2$.

\section{Consequences and questions}

If $A$ is a Cohen-Macaulay local ring, let $A E(A)$ denote the Grothendieck group of finite length $A$-modules of finite projective dimension. This is a special case of a notion studied in $\left[\mathrm{F}_{1}\right],[\mathrm{FFI}]$. There, when $A$ is not $C-M, A(A)$ is defined as the Grothendieck group of finite free complexes with finite length homology, under certain relations.

It was previously not known whether, for an arbitrary local ring $A, A E(A)$ would be generated by the classes of Koszul complexes of systems of parameters. In the case where $A$ is $C-M$, in terms of the definition via modules given above, this question becomes, is $A(A)$ generated by the classes $\left[A /\left(u_{1}, u_{2}, \ldots, u_{n}\right)\right]$ where $u_{1}, \ldots, u_{n}$ is a system of parameters (equivalently, a maximal $A$-sequence) in $A$. This is known to be true for Cohen-Macaulay local rings $A$ with $\operatorname{dim} A \leqq 2$ : the idea of the proof can be found in $\left[\mathrm{H}_{1}\right]$, although a complete argument for the general case does not seem to appear in detail in the literature.

By virtue of our example we can now assert:

(7.1) Proposition. Let $G$ be the quotient of $A(R)$ by the subgroup generated by the classes $\left[R /\left(u_{1}, u_{2}, u_{3}\right)\right]$, where $u_{1}, u_{2}, u_{3}$ is a system of parameters for $R$. Then if $M$ is a module of finite length and finite projective dimension such that

$$
\chi(M, R / P)= \pm 1
$$

then $[\mathrm{M}]$ is not torsion in $G$, and, in fact, $\mathbf{Z} \cdot[M]$ is a direct summand of $G$ as an abelian group ( $\mathrm{Z}$ is the integers).

Proof. Define

$$
h: \not E(R) \rightarrow \mathbf{Z}
$$

by $h([N])=\chi(N, R / P)$. By [L], $h$ kills each $\left[R /\left(u_{1}, u_{2}, u_{3}\right)\right]$, and so $h$ induces a map

$$
h: G \rightarrow \mathbf{Z} \cong \mathbf{Z} \cdot[M]
$$


such that $\bar{h}([M])= \pm 1$, i.e., $\bar{h}$ is surjective, which splits the inclusion $\mathbf{Z} \cdot[M]$ $\hookrightarrow$ G. Q.E.D.

We have no idea just how large $A(R)$ is. One intriguing question is this:

(7.2) Question. For modules $M$ of finite length and finite projective dimension of $R$, let

$$
\mu(M)=|\chi(M, R / P)| / /(M) .
$$

What is $\sup _{M} \mu(M)$, and is there a module $M$ for which the supremum is achieved?

Note that the original generalized Serre conjecture had $\mu(M)=0$, which is, of course, not true. For modules $M$ of finite length and finite projective dimension killed by $\left(x_{2}, x_{4}\right) m+\mathfrak{m}^{3}$, we know $\mu(M) \leqq 1 / 15$. We do know that

$$
0 \leqq \mu(M)<1
$$

for every $M$, and so we know

$$
1 / 15 \leqq \sup _{M} \mu(M) \leqq 1 .
$$

It would be interesting if $\mu=\sup _{M} \mu(M)$ is not achieved, for the following reason:

(7.3) Proposition. If $\sup _{M} \mu(M)$ is not achieved, then there exist finite length $R$ modules of finite projective dimension of arbitrarily great length possessing no proper nonzero submodules of finite projective dimension.

Proof. If the conclusion is false, then there exists a fixed positive integer $N$ such that every finite length module of finite projective dimension has a filtration in which every factor has finite projective dimension and length $\leqq N$.

For any such module $M$ of length $\leqq N,|\chi(M, R / P)| \leqq N$, and so there are only finitely many possibilities for $\chi(M, R / P)$, lying among the integers between $-(N-1)$ and $N-1$.

Let $\left(a_{1}, b_{1}\right), \ldots,\left(a_{r}, b_{r}\right)$ be the pairs of integers which can occur as $(\chi(M, R / P), l(M))$ with $l(M) \leqq N$. The $b_{i}$ are integers between 1 and $N$, while $\left|a_{i}\right|<b_{i}$. Then for a module $M$ with a filtration containing $k_{i}$ copies of factors which yield $\left(a_{i}, b_{i}\right), 1 \leqq i \leqq r$, we have

$$
\mu(M)=\left|\sum k_{i} q_{i}\right| / \sum k_{i} b_{i} \leqq \sum k_{i}\left|a_{i}\right| / \sum k_{i} b_{i}
$$

and since $\chi\left(M^{*}, R / P\right)=-\chi(M, R / P)$ there is no loss of generality in working only with pairs $\left(a_{i}, b_{i}\right)$ with $a_{i} \geqq 0$.

But if $b, b^{\prime}>0$ and $\frac{a}{b} \leqq \frac{a^{\prime}}{b^{\prime}}$, then $\frac{a+a^{\prime}}{b+b^{\prime}} \leqq \frac{a^{\prime}}{b^{\prime}}$. From this fact and a trivial induction we see that

$$
\sum k_{i} a_{i} / \sum k_{i} b_{i} \leqq \max _{i}\left\{a_{i} / b_{i}\right\} .
$$

Thus, the supremum is actually achieved on some module of length $\leqq N$. Q.E.D. 
If $M$ is finitely generated and has finite projective dimension over a Noetherian ring $S, N$ is finitely generated, and $M \otimes_{S} N$ has finite length, then we may consider the truncated Euler characteristics

$$
\chi_{i}(M, N)=\sum_{t}(-1)^{i} l\left(\operatorname{Tor}_{i+t}^{s}(M, N)\right), \quad 0 \leqq t \leqq p d M-i,
$$

of which $\chi_{0}(M, N)=\chi(M, N)$ is the foremost example. Until the advent of the examples constructed here, it seemed to be a viable conjecture that $\chi_{i}(M, N) \geqq 0$ whenever it is defined. This is true, for example, over unramified regular rings: see [L]. There it is shown that $\chi_{i}(M, N)=0, i \geqq 2$, implies $\operatorname{Tor}_{j}(M, N)=0, j \geqq i$. Recently, this was extended $\left[\mathrm{H}_{4}\right]$ to the case $i=1$ (but, still, only for unramified regular local rings).

We want to point out that the examples given here lead to examples where $\chi_{i}(M, N)<0$ even when $\operatorname{dim} S=2, i=1$.

The construction of these examples is based on our main counterexample and the following:

(7.4) Proposition. Let $A$ be a normal 3-dimensional Gorenstein local domain. Let $M$ be an A-module of finite length and finite projective dimension. Let $N$ be an $A$-module of depth 2 such that $N^{\prime}=\operatorname{Ext}^{1}(N, A)$ has finite length. Let ${ }^{\vee}$ denote the Matlis dual. Then

$$
\chi_{2}\left(M^{\vee}, N^{\prime}\right) \leqq \chi(M, N) .
$$

Proof. We first note that if $W$ has finite length we may identify its Matlis dual $W^{\vee}$ with $\operatorname{Ext}^{3}(W, A)$, a module of the same length. We also note that if $W$ has finite projective dimension and finite length then

i) $\operatorname{Ext}^{i}(W, V) \cong \operatorname{Tor}_{3-i}\left(W^{\vee}, V\right)$,

for if $P_{*}$ is a minimal projective resolution of $W$, then $\operatorname{Hom}\left(P_{*}, A\right)$ is a minimal projective resolution of $W^{\vee}$ and $\operatorname{Hom}\left(P_{*}, V\right)$, whose cohomology is $\operatorname{Ext}^{i}(W, V)$, may be identified with $\operatorname{Hom}\left(P_{*}, A\right) \otimes V$.

Let

ii) $W^{i j}=\operatorname{Ext}^{i}\left(M, \operatorname{Ext}^{j}(N, A)\right)$.

There are spectral sequences with $E^{2}$ terms $\operatorname{Ext}^{\varphi}\left(\operatorname{Tor}_{q}(M, N), A\right)$ and $W^{i j}$, respectively, which converge to (associated graded complexes of) the same $E^{\infty}$ complex. Since depth $N \geqq 2$, $\operatorname{Tor}_{q}(M, N)=0$ unless $q=0$ or 1 . Now $\operatorname{Tor}_{q}(M, N)$ has finite length (since $M$ does) so $\operatorname{Ext}^{P}\left(\operatorname{Tor}_{q}(M, N), A\right.$ ) vanishes $(A$ is Gorenstein) unless $p=3$. It follows that $E^{\infty}$ is 0 except when $p+q$ is 3 or 4 , in which case it coincides with $\operatorname{Ext}^{3}(M \otimes N, A)$ or $\operatorname{Ext}^{3}\left(\operatorname{Tor}_{1}(M, N), A\right)$, respectively.

Since $N$ has depth 2 and $A$ is Gorenstein, $\operatorname{Ext}^{j}(N, A)$ vanishes except for $j$ $=0,1$. The $W^{i j}$ then display the following pattern:

$$
\begin{array}{c|llcc}
1 & W^{10} & W^{11} & W^{12} & W^{13} \\
0 & W^{00}=0 & W^{01}=0 & W^{02} & W^{03} \\
\hline j / i & 0 & 1 & 2 & 3
\end{array}
$$

where all $W^{i j}$ not shown are 0 . The two zeros in the bottom row occur because $\operatorname{Hom}(N, A)$ is reflexive, hence of depth at least two, and so $\operatorname{Ext}^{i}(M, \operatorname{Hom}(N, A))=0$ for $i<2$. 
From the fact that the homology of the total complex here yields an associated graded complex of $E^{\infty}$ we now obtain, as usual, a long exact sequence:

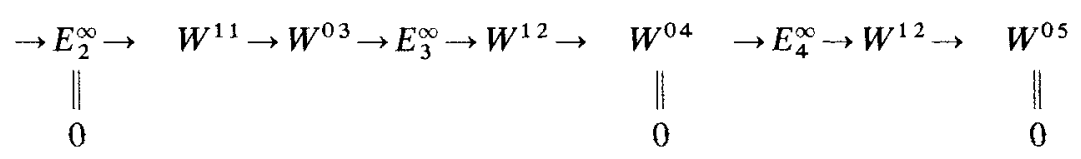

The first six terms give an exact sequence:

iii) $0 \longrightarrow \operatorname{Ext}^{1}\left(M, N^{\prime}\right) \longrightarrow \operatorname{Ext}^{3}(M, \operatorname{Hom}(N, A)) \longrightarrow \operatorname{Ext}^{3}(M \otimes N, A)$

$$
\stackrel{\alpha}{\longrightarrow} \operatorname{Ext}^{2}\left(M, N^{\prime}\right) \longrightarrow 0
$$

(where $N^{\prime}=\operatorname{Ext}^{1}(N, A)$ ) while the last four give

iv) $\operatorname{Ext}^{3}\left(\operatorname{Tor}_{1}(M, N), A\right) \cong \operatorname{Ext}^{3}\left(M, N^{\prime}\right)$.

Since $p d_{A} M=3$, since $M$ has finite length, and since depth $N=2$, we know that $\operatorname{Tor}_{i}(M, N)=0, i \geqq 2$. Thus,

v) $\chi(M, N)=l(M \otimes N)-l\left(\operatorname{Tor}_{1}(M, N)\right)$.

Now, $(M \otimes N)^{\vee} \cong \operatorname{Ext}^{3}(M \otimes N, A)$ and so

$$
l(M \otimes N)=l\left((M \otimes N)^{\vee}\right)=l\left(\operatorname{Ext}^{3}(M \otimes N, A)\right) .
$$

Thus, from the surjectivity of $\alpha$ in iii) we have

vi) $l(M \otimes N) \geqq l\left(\operatorname{Ext}^{2}\left(M, N^{\prime}\right)\right)$

Also,

$$
=l\left(\operatorname{Tor}_{1}\left(M^{\vee}, N^{\prime}\right)(\text { by } i)\right) \text {. }
$$

and so

$$
\begin{aligned}
& \operatorname{Tor}_{1}(M, N)^{\vee} \cong \operatorname{Ext}^{3}(\operatorname{Tor}(M, N), A) \\
& \cong \operatorname{Ext}^{3}\left(M, N^{\prime}\right) \quad \text { (by iv)) } \\
& \left.\cong M^{2} \otimes N^{\prime} \quad \text { (by i) }\right)
\end{aligned}
$$

vii) $l\left(\right.$ Tor $\left._{1}(M, N)\right)=l\left(\right.$ Tor $\left._{1}(M, N)^{\vee}\right)=\left(M^{\vee} \otimes N^{\prime}\right)$.

Together, v), vi) and vii) yield

viii) $\chi(M, N) \geqq l\left(\right.$ Tor $\left._{1}\left(M^{\vee}, N^{\prime}\right)\right)-l\left(M^{\vee} \otimes N^{\prime}\right)$.

Now, $M^{\vee}$ has finite projective dimension and finite length and $N^{\prime}$ $=\operatorname{Ext}^{1}(N, A)$ has finite length so that

$$
\begin{aligned}
\chi\left(M^{\vee}, N^{\prime}\right) & =0 \Rightarrow l\left(\operatorname{Tor}_{1}\left(M, N^{\prime}\right)\right)-l\left(M^{\vee} \otimes N^{\prime}\right) \\
& =l\left(\operatorname{Tor}_{2}\left(M^{\vee}, N^{\prime}\right)\right)-l\left(\operatorname{Tor}_{3}\left(M^{\vee}, N^{\prime}\right)\right) \\
& =\chi_{2}\left(M^{\vee}, N^{\prime}\right)
\end{aligned}
$$

which, together with viii), yields the desired inequality. Q.E.D.

(7.5) Remarks. We next want to discuss some implications of the existence of the counterexamples of Sect. 5 for questions about nonnegativity of $\chi_{i}$. It is not 
surprising that counterexamples to this nonnegativity are immediately forthcoming.

Specifically, consider an $R$-module $M$ with $l(M)=15, p d_{R} M<\infty$ and $\chi(M, R / P)=1$, where, as before, $P=\left(x_{1}, x_{2}\right)$. Since $m^{3} M=0, l\left(M / P^{t} M\right)=l(M)$ $=15$ for $t \geqq 3$. The exact sequence

$$
0 \rightarrow P^{t} \rightarrow R \rightarrow R / P^{t} \rightarrow 0
$$

yields

$$
0 \longrightarrow \operatorname{Tor}_{1}\left(M, R / P^{t}\right) \stackrel{\gamma}{\rightarrow} M \otimes P^{t} \stackrel{\beta}{\longrightarrow} M \stackrel{\alpha}{\longrightarrow} M / P^{t} M \longrightarrow 0
$$

where $\alpha$ is an isomorphism for $t \geqq 3$, and so $\beta$ is 0 and $\gamma$ is an isomorphism as well for $t \geqq 3$. It follows that

$$
\begin{aligned}
\chi_{1}\left(M, R / P^{t}\right) & =\chi\left(M, P^{t}\right)=\chi(M, R)-\chi\left(M, R / P^{t}\right) \\
& =l(M)-\chi\left(M, R / P^{t}\right) .
\end{aligned}
$$

Since $R_{P}$ is a $D V R, R / P^{t}$ has a prime filtration with $t$ factors $\cong R / P$ and all remaining factors of the form $R / q$, where $q \not P$. Since $R / q$ is a torsion-module over the regular ring $R / P$, its class in the Grothendieck group of all finitely generated $(R / P)$-modules is 0 , and this is also true in the Grothendieck group of all finitely generated $R$-modules. It follows that $\chi(M, R / q)=0$ for each such $q$, and hence

$$
\chi\left(M, R / P^{t}\right)=t \chi(M, R / P)=t
$$

whence $\chi\left(M, P^{t}\right)=l(M)-t=15-t$.

Hence, for $t \geqq 15, \chi\left(M, P^{t}\right) \leqq 0$ and $\chi\left(M, P^{t}\right)<0$ for $t \geqq 16$.

Note also that $\chi\left(M, P^{15}\right)=0=\chi_{1}\left(M, R / P^{15}\right)$ but

$$
\operatorname{Tor}_{1}\left(M, R / P^{15}\right) \cong M \otimes P^{15} \neq 0 .
$$

(7.6) Remarks. It is not hard to see that $P^{t}=P^{(t)}$ for all integers $t$. For this purpose, we briefly use $R$ to denote $K\left[X_{i}\right] /\left(X_{1} X_{4}-X_{2} X_{3}\right)$, without localizing, and $P=\left(X_{1}, X_{2}\right) R$. It suffices to see that $P^{t}=P^{(t)}$ here. The $K$-homomorphism

$$
K\left[X_{1}, X_{2}, X_{3}, X_{4}\right] \rightarrow K\left[u_{1}, u_{2}, v_{1}, v_{2}\right]=T
$$

which sends the entries of $\left[\begin{array}{ll}X_{1} & X_{2} \\ X_{3} & X_{4}\end{array}\right]$ to the corresponding entries of $\left[\begin{array}{ll}u_{1} v_{1} & u_{1} v_{2} \\ u_{2} v_{1} & u_{2} v_{2}\end{array}\right]$ has kernel $\left(X_{1} X_{4}-X_{2} X_{3}\right)$ which enables us to identity $R$ with the subring $K\left[u_{i} v_{j}\right] \subset T$. Then $P=\left(u_{1}\right) \cap R$ and $P^{(t)}=\left(u_{1}^{t}\right) \cap R$, and it is quite easy to see that

$$
\begin{aligned}
\left(u_{1}^{t}\right) \cap R & =\left(u_{1}^{t} v_{1}^{i} v_{2}^{j}: i+j=t\right) R \\
& =P^{t} R .
\end{aligned}
$$

Thus, $R / P^{t}=R / P^{(t)}$ has depth 1 , and so $P^{t}$ has length 2 as an $R$-module. Moreover, $P^{t}$ becomes free if we localize at any prime except $m$ $=\left(x_{1}, x_{2}, x_{3}, x_{4}\right)$, since $P R_{x_{i}}$ is principal, $i=1,2,3,4$.

We revert now to studying the local version of $R$. The remark above shows that for all $t, P^{t}$ has depth 2 and $\operatorname{Ext}^{1}\left(P^{t}, R\right)$ has finite length.

We now put this together with Proposition (7.4). 
(7.7) Proposition. For $t \geqq 3$ let

$$
N_{t}=\operatorname{Ext}^{1}\left(P^{t}, R\right),
$$

an R-module of finite length. Let $M$ be an $R$-module of length 15 and finite projective dimension such that

$$
\chi(M, R / P)=1
$$

For $t \geqq 16, \chi_{2}\left(M^{\vee}, N_{t}\right)<0$.

Proof. By $(7.4), \chi_{2}\left(M^{\vee}, N_{t}\right) \leqq \chi\left(M, P^{t}\right)=15-t<0$, using the information in Remarks (7.5), (7.6). Q.E.D.

(7.8) Proposition. With notation as in (7.7) let $M_{1}$ be a first module of syzygies of $M^{\vee}$, let $x \in \operatorname{Ann} N_{t}$ be any nonzero element of $R$, let $S=R / x R$ and suppose $t \geqq 16$. Then

$$
\chi_{2}^{R}\left(M^{\vee}, N_{t}\right)=\chi_{1}^{R}\left(M_{1}, N_{t}\right)=\chi_{1}^{S}\left(M_{1} / x M, N_{t}\right)<0 .
$$

Proof. $\operatorname{Tor}_{i}^{R}\left(M^{\vee}, N_{t}\right) \cong \operatorname{Tor}_{i-1}^{R}\left(M_{1}, N_{t}\right), i \geqq 2$, while

$$
\operatorname{Tor}_{i-1}^{R}\left(M_{1}, N_{t}\right) \cong \operatorname{Tor}_{i-1}^{S}\left(M_{1} / x M_{1}, N_{t}\right),
$$

all $i$, since $x$ is a nonzero divisor on $R, M_{1}$ and kills $N_{t}$. Q.E.D.

Thus we obtain the example of a two-dimensional local ring $S$, which is, in fact, a complete intersection, an $S$-module of finite projective dimension $\vec{M}$ $=M_{1} / x M_{1}$, and an $S$-module of finite length, $N_{t}$, with $\chi_{1}\left(\bar{M}, N_{t}\right)<0$.

(7.9) Remarks on rigidity. It remains an open question whether, if $p d M<\infty$ and $\operatorname{Tor}_{i}(M, N)=0$ then $\operatorname{Tor}_{j}(M, N)=0$ for $j \geqq i$. Here, $M, N$ are finitely generated modules over a Noetherian ring. So far as we know, this question, raised by $M$. Auslander in $\left[\mathrm{A}_{1}\right],\left[\mathrm{A}_{2}\right]$, is open even if $p d M=2$. It reduces to the case of local rings, even complete local rings, and is known if the ring is regular [L].

The examples given here suggest that this conjecture may be false. Our objective here is to present the simplest situation in which the question is still open in terms of a down-to-earth problem in linear algebra which will exhibit certain clear analogies with the problem solved earlier to give the counterexample for the generalized Serre conjecture on multiplicities.

What we want to do is consider a completely concrete form of the rigidity conjecture for modules $M$ with free resolutions of length 2 of the form

$$
0 \rightarrow A \rightarrow A^{b} \rightarrow A^{b} \rightarrow M \rightarrow 0, \quad b \geqq 2 .
$$

Although it is not necessary, we restrict attention to the case where $A$ contains field, for simplicity. In this situation one can show that it suffices to study a specific module $M$ over a certain "generically" constructed ring $A$, together with an arbitrary $A$-module $N$ of finite length. For more details about the generic rings and complexes we refer the reader to $\left[\mathrm{H}_{2}\right]$.

These generic rings over a fixed field $K$ are constructed as follows:

Let $U=\left[u_{1}, \ldots, u_{b}\right], V^{t}=\left[v_{1}, \ldots, v_{b}\right]$ so that (superscript $t$ denotes transpose) $U, V$ are $1 \times b$ and $b \times 1$ matrices of indeterminates and let $Z=\left(z_{i j}\right)$ be a $b$ 
$\times b$ matrix of indeterminates. Let

$$
A=K\left[u_{j}, v_{i}, z_{i j}\right] / J
$$

where $J$ is the ideal generated by $\operatorname{det} Z$ and the entries of the matrices $U Z, Z V$, and $V U-\operatorname{adj} Z$ (where adj denotes the classical adjoint, i.e., the transpose of the cofactor matrix). We write ${ }^{-}$to denote images modulo $J$.

Then, with matrices acting on the right,

$$
0 \longrightarrow A \stackrel{\sigma}{\longrightarrow} A^{b} \stackrel{z}{\longrightarrow} A^{b} \longrightarrow M \longrightarrow 0
$$

is exact, where $M$ is simply the cokernel of $\bar{Z}$. To give an $A$-module $N$ of finite length $n$, say $N=K^{n}$, is then the same as to give $b^{2}+2 b$ commuting $n \times n$ matrices corresponding to the $u_{j}, v_{i}, z_{i j}$, which satisfy the conditions which define $J$.

Let $\mathscr{U}, \mathscr{Z}$, respectively, be the $n$ by $b n$ and $b n \times b n$ matrices obtained by replacing the entries of $\bar{U}, \bar{Z}$ by the corresponding $n \times n$ matrices which give the actions of the entries on $N=K^{n}$.

The rigidity conjecture then asserts that

$$
\operatorname{rank} \mathscr{U}+\operatorname{rank} \mathscr{Z}=n b \Rightarrow \operatorname{rank} \mathscr{U}=n .
$$

An equivalent assertion is that

$$
\operatorname{rank} \mathscr{U}+\operatorname{rank} \mathscr{Z}=n b \Rightarrow \operatorname{rank} \mathscr{Z} \leqq n(b-1) .
$$

We do not know whether this is true even when $b=3$. When $b=2$ the assertion rank $\mathscr{Z} \leqq n$ is hard to give counterexamples to even under the mild hypotheses that the entries of $\mathscr{Z}$ commute and yield $(2 \times 2)$ determinant 0 : one needs $n \geqq 8$ and the smallest example we know of is the counterexample of Sect. 5, with $n=15$. Of course, in the situation of rigidity there is much more hypothesis (in particular, the condition $\bar{V} \bar{U}=\operatorname{adj} \bar{Z}$, which, for $b=2$, does imply rank $\mathscr{Z} \leqq n$ ).

(7.10) Remarks on the Gothendieck group of $R$. We want to note here that the Grothendieck group $G$ of $R$ is the free abelian group on the generators $[R]$ and $[R / P]$, and observe some consequences of this fact.

To see that these two generate we note that it suffices to prove this instead where $R=K\left[X_{i}\right] /\left(X_{1} X_{4}-X_{2} X_{3}\right)$ (not localized), and $P=\left(X_{1}, X_{2}\right) R$. Every elements of $G$ is equivalent, modulo $[R]$, to each of its modules of syzygies. Since $R_{x_{i}}$ is regular, these are eventually projective and hence free, for $R_{x_{1}} \cong K\left[X_{1}, X_{2}, X_{3}\right]_{X_{1}}$. Let $M$ be torsion-free such that $M_{x_{1}}$ is free. Then we can embed $M \rightarrow R^{k}$ for some $k$ such that the cokernel is annihilated by a power of $x_{1}$. Thus, modulo [R],M is equivalent to the sum of the factors in a prime filtration of the cokernel. Each is a prime containing $x_{1}$, and so contains either $x_{2}$ or $x_{3}$ and hence may be regarded as a module over the regular ring $R /\left(x_{1}, x_{j}\right), j=2$ or $3,=K\left[x_{4}, x_{5-j}\right]$, and so has a finite free resolution over $R /\left(x_{1}, x_{j}\right)$. It follows that $G$ is generated by $[R],[R / P],[R / Q]$, where $Q$ $=\left(x_{1}, x_{3}\right)$. But $\left.0 \rightarrow R \stackrel{x_{1}}{\longrightarrow} Q \rightarrow R / P \rightarrow 0\right)$ is exact, showing that $[Q]=[R / P]$ $+[R]$, which implies that $[R / Q]=-[R / P]$. 
Now, the additive map given by torsion-free rank is 1 on $[R]$ and 0 on $[R / P]$, while if $M$ is the example of Sect. 5 with $p d M<\infty, l(M)=15, \chi(M, R / P)$ $=1$, then the additive map $\alpha(N)=\chi(M, N)-15 r k N$ is 0 on $N=R, 1$ on $N$ $=R / P$. It follows that $G$ is the free abelian group with generators $[R],[R / P]$, and that for all $N$

$$
[N]=(r k N)[R]+(\chi(M, N)-15 r k N)[R / P] .
$$

This shows that for any module $M_{1}$ of finite length and finite projective dimension, the behavior of $\chi\left(M_{1}, N\right)$ for all finitely generated modules $N$ is completely determined by the behavior of $\chi\left(M_{1}, R\right)=l\left(M_{1}\right)$ and $\chi\left(M_{1}, R / P\right)$.

We also note that for such a module $M_{1}, \chi\left(M_{1}, N\right)=0$ for $\operatorname{dim} N \leqq 1$ : see $\left[\mathrm{D}_{1}\right]$, Proposition (1.3).

(7.11) Remarks. Let $S$ be a local ring, $N$ a finitely generated $S$-module of dimension $d$ with $p d_{S} N<\infty$, and let $P$ be a prime ideal of $S$ such that $N / P N$ has finite length and $\operatorname{dim} N+\operatorname{dim} S / P \leqq \operatorname{dim} S$. Let $e(P, N)$ denote $d ! a$, where $a$ is the coefficient of the degree $d$ term of the Hilbert polynomial which agrees with $l\left(N / P^{n} N\right)$ for large $n$.

If $S$ is an equicharacteristic or, more generally, unramified regular local ring then, by $\left[\mathrm{D}_{3}\right]$, Theorem (2.3):

$$
\chi(N, R / P) \leqq e(P, N) .
$$

We note here that this fails over the local ring $S=R$ we have been studying, with $P=\left(x_{1}, x_{2}\right) R$ as usual, for a class of modules $N$ with $\operatorname{dim} N=1$, depth $N$ $=0$ and $p d_{R} N<\infty$.

To see this choose any module $N_{0}$ of dimension one with $p d N_{0}<\infty$ such that $N_{0} / P N_{0}$ has finite length. For example, we may choose any $R$-sequence $u_{1}, u_{2}$ which is also an $(R / P)$-sequence and let $N_{0}=R /\left(u_{1}, u_{2}\right)$. Let $M$ be of finite length and finite projective dimension over $R$ with $\chi(M, R / P)>0$. Let $N_{k}$ $=N_{0} \oplus M^{k}$. Then

$$
\psi\left(N_{k}, R / P\right)=\chi\left(N_{0}, R / P\right)+k \chi(M, R / P)
$$

which can be made arbitrarily large by choosing $k$ large, while

$$
e\left(P, N_{k}\right)=e\left(P, N_{0}\right)
$$

because $l\left(M^{k} / P^{n} \cdot M^{k}\right)=l\left(M^{k}\right)$ for all sufficiently large $n$, and so adding $M^{k}$ has no effect on the degree one term of the Hilbert polynomial of $N_{0}$. Evidently, then,

$$
\chi\left(N_{k}, R / P\right)>e\left(P, N_{k}\right)
$$

for all sufficiently large $k$.

(7.12) Remarks. Let $(M, N)$ be a pair of finitely generated modules over a Gorenstein local ring $S$ of positive prime characteristic $p$ such that $p d_{S} M<\infty$, $l\left(M \otimes_{S} N\right)<\infty$, and $\operatorname{dim} M+\operatorname{dim} N \leqq \operatorname{dim} S$. Then we can define a "multiplicity" function in this situation as follows (see $\left[\mathrm{D}_{4}\right]$ for details):

$$
\chi_{\infty}(M, N)=\lim _{e \rightarrow \infty} \chi\left(F^{e}(M), N\right) / p^{e \operatorname{codim} M}
$$


where $F^{e}(M)$ denotes the $e$ th iterated Frobenius functor of Peskine-Szpiro: see [PS ${ }_{1}$ ]. (If $M=\operatorname{coker}\left(a_{i j}\right), F^{e}(M)=\operatorname{coker}\left(a_{i j}^{q}\right)$, where $q=p^{e}$.)

$\chi_{\infty}$ behaves better than $\chi$ in some ways: by $\left[\mathrm{D}_{4}\right]$, we have

i) $\chi_{\infty}(M, N)=0$ if $\operatorname{dim} M+\operatorname{dim} N<\operatorname{dim} \mathrm{S}$,

ii) if $M$ is Cohen-Macaulay and $\operatorname{dim} M+\operatorname{dim} N=\operatorname{dim} S$ then $\chi_{\infty}(M, N)>0$; in fact in this case

$$
\chi_{\infty}(M, N)=\lim _{e \rightarrow \infty} l\left(F^{e}(M) \otimes N\right) / p^{e \operatorname{codim} M} .
$$

See $\left[\mathrm{D}_{4}\right]$ for details.

We note here that for $S=R$ we can choose $M, N$ as above with $\operatorname{dim} M=1$, depth $M=0$ such that

$$
\chi_{\infty}(M, N)<0 .
$$

In fact take $N=R / P$, let $u_{1}, u_{2}$ be an $R$-sequence whose residues modulo $P$ form a maximal $R$-sequence in $R / P$ and let

$$
M_{k}=R /\left(u_{1}, u_{2}\right)+M_{0}^{k}
$$

where $M_{0}$ has finite length, finite projective dimension and $\chi\left(M_{0}, R / P\right)<0$. For large $k$ we then have

But:

$$
\chi\left(M_{k}, R / P\right)=0 .
$$

(7.13) Lemma. Let $M$ be any finitely generated module over $R$ of dimension one with $p d_{R} M<\infty$ such that $l(M / P M)$ is finite. Then

$$
\chi_{\infty}(M, R / P)=\chi(M, R / P) .
$$

Proof. After a change of fields we may assume that $K$ is algebraically closed. Given an $R$-module $N$, let ${ }^{e} N$ denote the result of restricting scalars to the first copy of $R$ under the $e^{\text {th }}$ iterate $F^{e}$ of Frobenius, $F^{e}: R \rightarrow R$. Then

$$
\chi\left(F^{e}(M), N\right)=\chi\left(M,{ }^{e} N\right)
$$

(see $\left[\mathrm{D}_{4}\right]$ ). Since $K$ is algebraically closed, if $N=R / P$, which is regular local of dimension $2,{ }^{e} N$ is the direct sum of $p^{2 e}$ copies of $R / P$, whence

$$
\chi\left(F^{e}(M), N\right)=p^{2 e} \chi(M, N)
$$

and the result is now immediate from the definition of $\chi_{\infty}$. Q.E.D.

Applying this with $M=M_{k}$ we see that

$$
\chi_{\infty}\left(M_{k}, R / P\right)=\chi(M, R / P)<0
$$

for all sufficiently large $k$.

\section{References}

[A $A_{1}$ Auslander, M.: Modules over unramified regular local rings. Illinois J. Math. 5, 631-645 (1961) 
[ $\left.\mathrm{A}_{2}\right]$ Auslander, M.: Modules over unramified regular local rings. Proc. Intern. Congress of Math., 1962, pp. 230-233

$\left[D_{1}\right]$ Dutta, S.P.: Generalized intersection multiplicities of modules. Trans. Amer. Math. Soc. 276, 657-669 (1983)

$\left[\mathrm{D}_{2}\right]$ Dutta, S.P.: Weak linking and multiplicities. J. Pure and Applied Algebra 27, 111-130 (1983)

$\left[\mathrm{D}_{3}\right]$ Dutta, S.P.: Symbolic powers, intersection multiplicity, and asymptotic behavior of Tor. J. London Math. Soc. (2) 28, 261-281 (1983)

[D4] Dutta, S.P.: Frobenius and multiplicities. J. of Algebra 85, 424-448 (1983)

$\left[\mathrm{D}_{5}\right]$ Dutta, S.P.: Generalized intersection multiplicities of modules II. Proc. A.M.S. (in press) (1984)

[DHM] Dutta, S.P., Hochster, M., McLaughlin, J.E.: Appendix to "Modules of Finite Projective Dimension with Negative Intersection Multiplicities," manuscript available from the authors

[E] Eisenbud, D.: Homological algebra on a local complete intersection, with an application to group representations. Trans. Amer. Math. Soc. 260, 35-64 (1980)

$\left[\mathrm{F}_{1}\right] \quad$ Fossum, R.: A-theory. Preprint Series, Politecnico di Torino, 1977

$\left[\mathrm{F}_{2}\right]$ Fossum, R: Correspondence, 1983

[FFI] Fossum, R., Foxby, H.-B., Iversen, B.: Characteristic classes of complexes. Preliminary preprint

[H $\left.\mathrm{H}_{1}\right]$ Hochster, $\mathrm{M}$ : : Cohen-Macaulay modules. Conference on Commutative Algebra. Lecture Notes in Math. Vol. 311, pp. 120-152. Berlin-Heidelberg-New York: Springer 1973

$\left[\mathrm{H}_{2}\right]$ Hochster, M.: Topics in the homological theory of modules over commutative rings, C.B.M.S. Regional Conference Series in Math., Vol. 24. Amer. Math. Soc., Providence, R.I., 1975

$\left[\mathrm{H}_{3}\right]$ Hochster, $\mathrm{M}$ : The local homological conjectures. In: Commutative Algebra: Durham 1981. Cambridge Univ. Press, London Math. Soc. Lecture Note Series. Vol. 72, pp. 32-54 (1982)

$\left[\mathrm{H}_{4}\right]$ Hochster, M.: Euler characteristics over unramified regular local rings. Illinois J. Math. in press (1984)

[L] Lichtenbaum, S.: On the vanishing of Tor in regular local rings. Illinois J. Math. 10, 220$226(1966)$

[M] Malliavin-Brameret, M.-P.: Une remarque sur les anneaux locaux réguliers, Sém. DubreilPisot (Algèbre et Théorie des Nombres), 24 année, 1970/71, $\mathrm{n}^{\circ} 13$

[PS $\left.{ }_{1}\right]$ Peskine, C., Szpiro, L.: Dimension projective finie et cohomologie locale. Inst. Hautes Etudes Sci. Publ. Math. 42, 323-395 (1973)

[PS $\left.{ }_{2}\right]$ Peskine, C., Szpiro, L.: Syzygies et multiplicités. C.R. Acad. Sci. Paris Ser. A-B 278, 1421-1424 (1974)

[S] Serre, J-P.: Algèbre locale. Multiplicités. Lecture Notes in Math. Vol. 11, 3éme édition. Berlin-Heidelberg-New York: Springer 1975

[W] Weil, A.: Foundations of algebraic geometry. Amer. Math. Soc. Colloq. Publ. Vol. 29, Amer. Math. Soc., Providence, R.I., Revised ed., 1962

Oblatum 16-VI-1984

\section{Note added in proof}

Independently, P. Roberts ( $n$ the vanishing of intersection multiplicities of perfect complexes, preprint 1984) and H. Gillet and C. Soulé ( $K$-théorie et nullité des multiplicités d'intersection, to appear in C.R. Acad. Sci. Paris) have shown that if $R$ is a local complete intersection, $\operatorname{dim} M$ $+\operatorname{dim} N<\operatorname{dim} R$ and both $M, N$, have finite projective dimension, then $l(M \otimes N)<\alpha$ implies $\chi(M, N)=0$. P. Roberts has also done the case where $R$ has an isolated singularity. Both papers include the use where $R$ is a possibly remified regular local ring. 